\title{
Approximate Aeroelastic Modeling of Flapping Wings: Comparison with CFD and Experimental Data
}

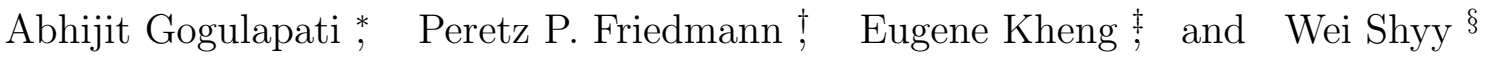 \\ Department of Aerospace Engineering, The University of Michigan, Ann Arbor, 48109, USA
}

\begin{abstract}
Results generated from an aeroelastic model obtained by coupling a nonlinear structural dynamic model based on MARC with an approximate aerodynamic model that incorporates leading edge vortices and a wake model are presented. The aerodynamic model, used in our earlier studies, is extended to forward flight. Results presented describe structural dynamic and aeroelastic studies conducted on isotropic and anisotropic wings in hover. For the cases considered, the approximate model shows reasonable agreement with the CFD based results. Comparisons with experiment indicate that the approximate model captures trends accurately, but under predicts the magnitude of thrust. Preliminary results obtained for a rigid flapping wing in forward flight indicate that, for the cases considered, peak lift generated by the wing increases as forward flight speed increases.
\end{abstract}

\section{Nomenclature}

\begin{tabular}{ll}
$A_{1}-A_{11}$ & Coefficients that are computed from the airfoil degrees of freedom \\
$\mathbb{C}$ & Complex plane \\
$C S 1$ & Cylindrical surface normal to the stroke plane; defined by motion of the airfoil \\
$C_{L}$ & Skewed cylindrical wake surface \\
$C_{T}$ & Lift coefficient \\
$c_{r}$ & Thrust coefficient; for helicopters, $C_{T}=\frac{T}{\rho_{\infty} \pi R_{s p a n}^{2}\left(\Omega R_{s p a n}\right)^{2}}$ \\
$\tilde{d}$ & Root chord \\
$E$ & Distance between a vortex and a point on the airfoil; \\
$\left(\hat{e}_{r}, \hat{e}_{\phi}\right)$ & measured along the composite airfoil wake surface \\
$f$ & Young's modulus \\
$h$ & Radial and angular unit vectors, shown in Figure 7 \\
$j, k$ & Flapping frequency \\
$L$ & Plunge degree of freedom of the airfoil \\
$l$ & Span station number \\
$M$ & Lift \\
$N_{\text {sections }}$ & Lead-lag degree of freedom of the airfoil \\
$N_{\theta}$ & User defined number of sections \\
$n_{\text {wssubit }}$ & Total number of aerodynamic span stations on the wing \\
$p_{\text {applied }}$ & Discretization of the circle \\
$p_{\text {computed }}$ & Number of wake sub-iterations \\
$p_{\text {limit }}$ & Pressure that is applied on the wing \\
$R_{j}$ & Pressure that is computed using the unsteady Bernoulli principle \\
$R_{\text {span }}$ & An upper bound value for pressure \\
\hline
\end{tabular}

\footnotetext{
*Ph.D. candidate, Student Member AIAA.

${ }^{\dagger}$ François-Xavier Bagnoud Professor, Fellow AIAA.

$\ddagger_{\text {Graduate Student. }}$

$\S$ Clarence "Kelly" Johnson Professor, Fellow AIAA.
} 


$\begin{array}{ll}R & \text { Radial coordinate of a point on the circle in the complex plane } \\ \Delta R_{j k} & \text { Distance between points on sections } C S 1_{j} \text { and } C S 1_{k} \text { that have the same } \\ & \text { angular coordinate } \theta \text { on the circle in the complex plane, shown in Figure } 8 \\ r_{c} & \text { Vortex core radius } \\ T & \text { Thrust } \\ t & \text { Time } \\ t_{h} & \text { Wing thickness } \\ U_{t i p} & \text { Maximum tip speed } \\ u_{\infty}, v_{\infty} & \text { Components of the free stream velocity resolved parallel and normal to the stroke plane } \\ \mathbf{V}_{\infty} & \text { Free stream velocity vector } \\ V_{\infty} & \text { Magnitude of free stream velocity } \\ v_{i} & \text { Inflow velocity through the rotor disk or stroke plane } \\ \Delta v_{x}^{f l e x}, \Delta v_{y}^{f l e x} & \text { Incremental components of velocity due to airfoil flexibility, } \\ & \text { measured in the airfoil fixed } x z \text { coordinate system } \\ v_{\theta} & \text { Tangential component of fluid velocity on the circle } \\ \mathbb{X} & \text { Cartesian coordinate system } \\ (X, Y, Z) & \text { Cartesian coordinate system; subscript indicates original location, } \\ \left(X, Y_{S P}, Z_{S P}\right) & \text { left superscript indicates projection, see Figure } 4 \\ \tilde{y}, \tilde{z} & \text { Coordinate system fixed to the stroke plane, shown in Figure } 4 \\ Z_{v} & \text { Coordinates of the center of a section of the airfoil-wake surface, Figure } 4 \\ z_{n w} & \text { Height of the near wake region, shown in Figure } 5\end{array}$

\section{Greek Symbols}

$\bar{\alpha} \quad$ Pitch angle

$\alpha_{R} \quad$ Angle between the tip path plane and the free stream velocity vector

$\beta \quad$ Flap angle

$\beta_{S P} \quad$ Angle between the stroke plane and the free stream velocity vector

$\beta_{0} \quad$ Flap amplitude

$\chi_{c s 2}, \chi_{\text {трР }} \quad$ Skew angle

$\phi \quad$ Angular position of the vortex on $C S 1$ or $C S 2$

$\varphi \quad$ Sweep angle of the instantaneous position of the feathering axis of the wing

$\epsilon_{\Gamma} \quad$ Circulation limit

$\varepsilon \quad=(\tau-\imath \sigma) / R$

$\left.\gamma_{0}\right|_{f s} \quad$ Component of vorticity due to free stream

$\left.\gamma_{0}\right|_{\text {us }} \quad$ Component of vorticity due to airfoil velocities

$\left.\gamma_{1}\right|_{l e v+w k} \quad$ Component of vorticity due to shed vortices

$\mathbf{d} \boldsymbol{\Gamma}_{v} \quad$ Circulation vector of a vortex

$d \Gamma_{v} \quad$ Magnitude of circulation of a vortex

$\theta \quad$ Angular coordinate on the circle in the complex plane

$\theta_{1} \quad$ Angle between $\tilde{d}$ and $\Delta R_{j k}$, shown in Figure 8 .

$\tau, \sigma \quad$ Thickness and camber parameters in the aerodynamic formulation

$\lambda \quad$ Inflow ratio

$\mu \quad$ Advance ratio

$\mu_{\infty} \quad$ Viscosity of the free stream

$\nu \quad$ Poisson's ratio

$\Omega, \omega \quad$ Circular frequency

$\rho \quad$ Density of the material

$\rho_{\infty} \quad$ Free stream density of the fluid

Additional Subscripts and Superscripts

$(\cdot)_{j},(\cdot)_{k} \quad$ Span station number

$p(\cdot) \quad$ Projected quantity

$(\cdot)_{v} \quad$ Property of a generic vortex

$\tilde{z}(\cdot) \quad$ Projection onto the $z=\tilde{z}$ plane 


\section{Introduction}

During the last decade there has been considerable interest in micro air vehicles (MAVs) for both military and civilian missions that involve confined spaces, such as buildings, or short distances. These vehicles typically have maximum geometric dimensions of $15 \mathrm{~cm}$, maximum weight of $100 \mathrm{grams}$, and are expected to operate at low Reynolds number $\left(10^{2}<\operatorname{Re}<10^{5}\right)$ and low forward flight speed $(<15 \mathrm{~m} / \mathrm{s}) .{ }^{1}$ In particular, flapping wing designs, which are inspired by hover-capable biological flyers such as insects, bats, and hummingbirds, have received considerable attention due to the exceptional flight capabilities observed in the biological counterparts. ${ }^{1}$

A significant portion of the research on flapping wing vehicles has focused on understanding the mechanisms that generate unsteady aerodynamic forces. This research ${ }^{1-6}$ has identified leading edge vortices (LEVs), wake capture, and tip vortices, as the primary force generating mechanisms. Attempts to model the aerodynamic environment in a quantitative manner have been based on two approaches: (1) computational fluid dynamics (CFD) simulations based on the solution of the Navier Stokes (NS) equations and (2) approximate aerodynamic models based on potential flow solutions. Simulations using CFD yield the best resolution of the unsteady flow field. However, such approaches require significant amounts of computer time and are expensive when conducting parametric studies. Approximate aerodynamic models offer a compromise between accuracy and computational efficiency and thus are suitable for trend and design studies. It is important to emphasize that the approximate models that have practical applications have to be able to model the effect of LEVs and wake capture.

The approximate unsteady aerodynamic theories used for flapping wing problems can be classified as assumed (or prescribed) wake and free wake models. Assumed wake models are classical unsteady models such as Theodorsen's theory. ${ }^{7}$ Reference [8] incorporated the effect of the LEVs in Theodorsen's theory by modifying the unsteady aerodynamic lift and moment expressions using the Polhamus leading edge suction analogy. ${ }^{9}$ This model $^{8}$ was compared to experiments and was capable of predicting the trends in aerodynamic forces.

Free wake models account for evolution of the wake, thereby providing a reasonable approximation to the development of the unsteady wake during a flapping cycle. In particular, free wake models that account for LEVs are two-dimensional formulations that use a discrete vortex representation of the wake. ${ }^{10-12}$ These formulations are suitable for flapping wings in hover following simplifying assumptions on the geometry of the shed wake. The model developed in Ref. [10], which accounts for separation close to the leading edge, compared well with experimental data for airfoils in steady flow. In this approach the chordwise location of the separation point, which may be obtained using independent computations or experiments, is explicitly incorporated into the formulation. Reference [11] presented the development of a vortex blob based formulation that simulates the unsteady flow field around flexible thin airfoil that is undergoing prescribed rigid body motion as well as prescribed deformation. The model displayed good correlation with previously published data for rigid airfoils; however, the study indicated that further investigation was required before the formulation could be used for aeroelastic studies. References $[12,13]$ presented the development and implementation of a discrete vortex model that is applicable to insect-like flapping wings in hover. The model was used to simulate rigid wings undergoing insect-like kinematics, and for the limited number of cases considered, compared well with experimental data. ${ }^{13}$

For the case of forward flight, the presence of a free stream alters the shed wake geometry and the subsequent wing-wake interaction in a significant manner. Therefore, additional modifications are needed before such two dimensional formulations ${ }^{10-12}$ can be used to model forward flight. Based on the review of the literature it appears that free wake models that incorporate LEVs have not been considered for flapping wings in forward flight.

The importance of wing flexibility in enhancing the lift producing capability of flapping wings has been considered in a number of studies. Studies that have attempted to examine this issue in a systematic manner include Refs. [8,14-19]. An important finding of Refs. [14,15], which was later corroborated by experiments conducted in Ref. [8], was that a dominant component of the loading on flapping wings is due to inertia loads. Reference [8] considered wing models based on membranes reinforced by metal beams. The aerodynamic loads were obtained by Theodorsen's theory modified using Polhamus analogy. This study, which considered wing flexibility in a linear manner, concluded that wing flexibility altered aerodynamic loads and therefore 
cannot be neglected. Reference [16], which considered a linear finite element model of a hawkmoth wing that was combined with CFD based flow field description, reported that spanwise flexibility had a favorable impact on thrust generated and power consumed by the flapping wing. Reference [17, 20] described a nonlinear aeroelastic model that is obtained by coupling a nonlinear finite element model of the wing with an approximate model. These studies, which examined representative MAV wings undergoing prescribed motion noted that for the cases considered, effect of aerodynamic loads was small compared to effect of inertia loads, that wing flexibility had a small but favorable impact on lift generation. References [18, 19] describe the development of a computational aeroelastic framework obtained by combining geometrically nonlinear beam and shell based structural dynamic models with a CFD based flow field. These studies, which examined isotropic low aspect ratio wings undergoing prescribed plunge motion, reported that flexibility increased lift and thrust generation for the range of kinematics considered.

The overall objective of this research is to develop an understanding of the effect of flexibility on the performance of anisotropic flapping wings in hover and forward flight. To achieve this objective the aeroelastic model described in Refs $[17,20]$ is employed for the case of hover. The aeroelastic model is based on a nonlinear FE model of the wing that is based on MSC MARC ${ }^{21}$ combined with the approximate unsteady aerodynamic model that was originally developed in Refs $[12,13]$. The specific objectives of the paper are:

1. Extend the aerodynamic formulation to the case of forward flight.

2. Verify the structural dynamic model by comparison with experimental data.

3. Compare results obtained using the approximate aeroelastic model with CFD and/or available experimental data for flapping wings in hover.

4. Examine the effect of a forward flight on forces generated by rigid flapping wings.

\section{Nonlinear Aeroelastic Model}

The aeroelastic model is obtained by coupling a nonlinear finite element model of the wing with an approximate aerodynamic model that incorporates LEVs and a free wake; a detailed description of the individual components and formulation of the aeroelastic equations of motion for flapping wings in hover was presented in Refs $[17,20]$. In this section, a summary of the aeroelastic model including modifications to the aerodynamic formulation to incorporate flapping wings in forward flight are presented.

\section{A. Structural Dynamic Model and Wing Kinematics}

The structural dynamic models of representative MAV wings are developed in MARC ${ }^{21}$ using shell elements that are capable of undergoing large amplitude rigid body motion as well as moderate-to-large flexible deformation. A variety of constitutive laws are available so that isotropic as well as anisotropic wings may be constructed. Wing kinematics, which consist of large amplitude rigid body rotations prescribed at the root, are applied as displacement boundary conditions at one or more nodes.

\section{B. Approximate Aerodynamic Model}

The unsteady aerodynamic loads generated on representative MAV wings are obtained using an approximate model that was developed in Refs $[12,13]$. This formulation, originally derived for the case of hover, is based on two-dimensional potential flow and uses a vorticity/circulation approach to compute the aerodynamic loads. The model was modified to account for spanwise and chordwise flexibility of flapping wings as described in Refs $[17,20]$. In the current paper, the aerodynamic formulation is extended to incorporate forward flight; details of the derivation along with an outline of overall approach are presented next.

\section{Outline of the Aerodynamic Formulation}

The overall approach, which retains the essential aspects of the hover formulation, ${ }^{12}$ is summarized in Figure 1. First, the wing is divided into several spanwise stations, as shown in Figure 2, where each section is represented as an airfoil. For each airfoil, an airfoil-wake surface that facilitates convenient definition of the airfoil degrees of freedom (DOF), and provides a reasonable approximation to the geometry of the 
shed wake, is identified. Subsequently, the airfoil and the airfoil-wake surface are transformed to a circle on a complex plane via a conformal mapping; consequently, the airfoil bound and shed wake vorticity are computed on the complex plane. The quasi-steady component of vorticity is obtained by neglecting the effect of the shed wake. The strength of shed vorticity is computed by enforcing a stagnation condition at the leading edge (LE) and a Kutta condition at the trailing edge (TE). The airfoil bound vorticity is obtained as a sum of the quasi-steady and wake-induced vorticity on the airfoil. Next, the vorticity on the complex plane is transformed back to the airfoil-wake surface using an inverse transform. Subsequently, the unsteady loads acting on the airfoil are obtained from the total vorticity either using the vortex impulse method, which yields the integrated force and moment, or the unsteady Bernoulli equation, which yields pressure. Finally, the shed vorticity is convected using the Rott-Birkhoff equation, which is derived from Biot-Savart law for two dimensional flow.
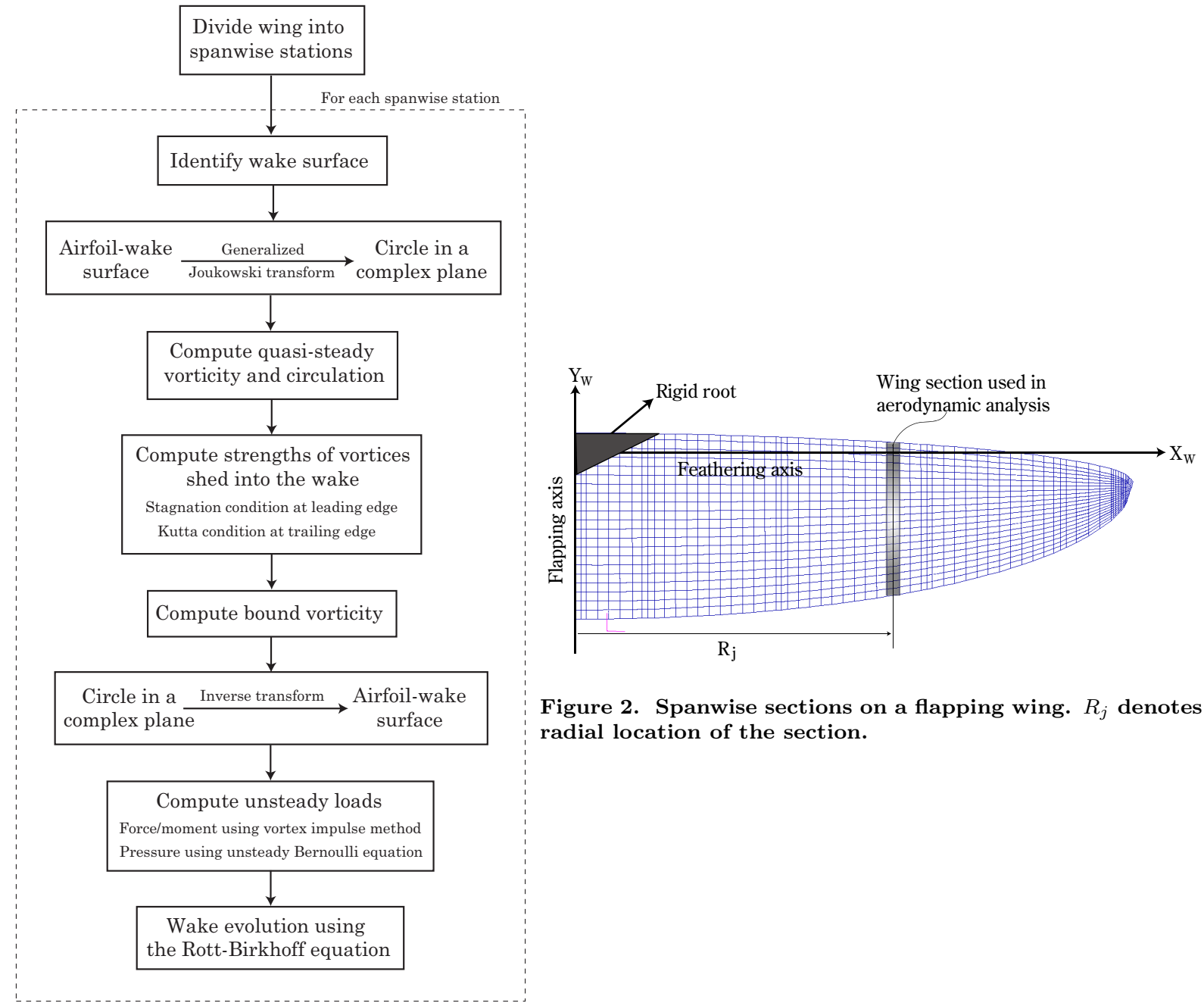

Figure 2. Spanwise sections on a flapping wing. $R_{j}$ denotes radial location of the section.

Figure 1. Schematic of the aerodynamic formulation.

\section{Definition of the Airfoil-Wake Surface}

Wing kinematics of biological flapping wing flyers, in both hover and forward flight, consists of a predominant sweep or flap motion in the stroke plane (SP), pitching about the feathering axis, and a comparatively small elevation angle. ${ }^{1,22}$ Therefore, the feathering axis of the wing is assumed to move on the stroke plane (SP). ${ }^{12}$ Consequently, the surface described by the airfoil motion is a cylinder that is normal to the stroke plane; this surface is labelled as $C S 1_{j}$ in Figure 4, For the case of hover, ${ }^{12}$ the shed wake is assumed to be confined to $C S 1_{j}$; therefore, $C S 1_{j}$ is a convenient choice for the airfoil-wake surface. For the case of forward flight, 


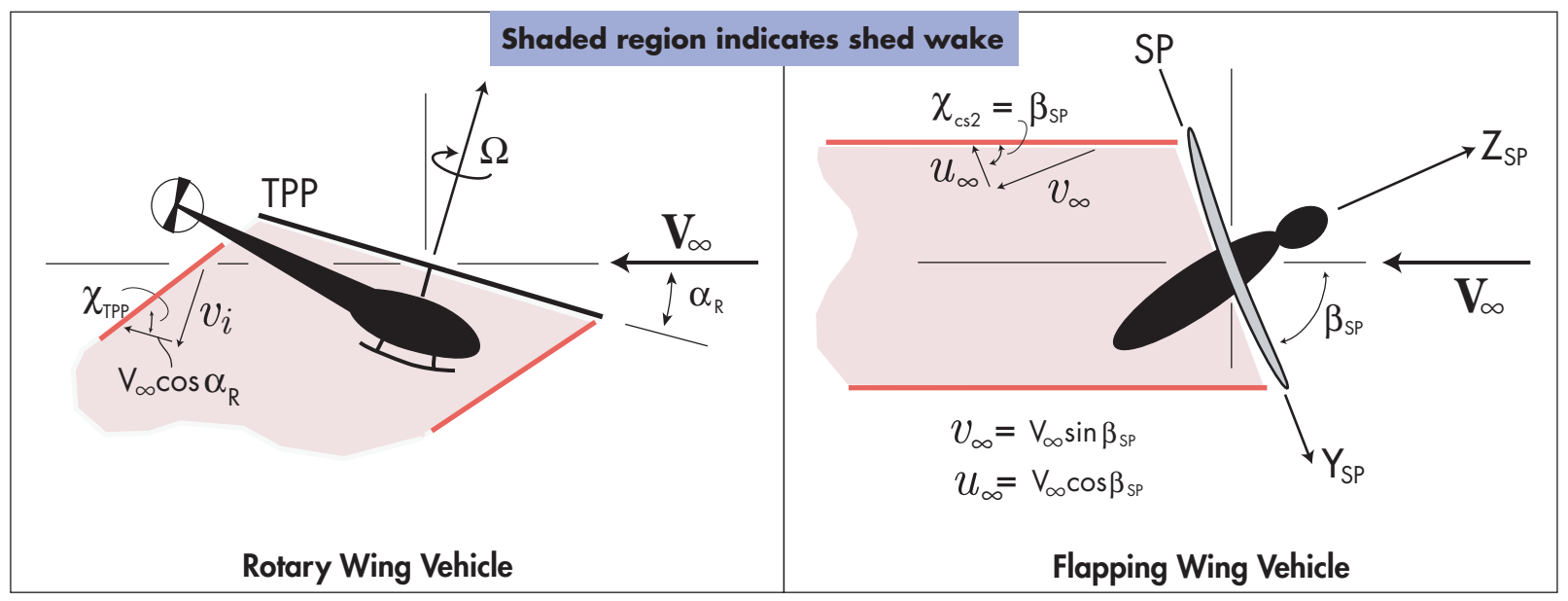

Figure 3. Shed wakes of rotary and flapping wing vehicles.

the vortices shed into the wake are carried away with the free stream due to velocity of forward flight; consequently, the wake is no longer confined to $C S 1_{j}$. Therefore, a suitable airfoil-wake surface has to be identified.

Chapters 12 and 14 of Ref [1] present rough comparisons between rotating propeller-type wings and flapping wings due to perceived similarities in the aerodynamic environments. Specifically, similarities such as the spanwise varying free stream velocity, overall geometry of the shed wake in hover, and presence of LEVs, are highlighted in Chapter 12. Chapter 14, which examined LEV formation and behavior at high angles of attack, concluded that both rotating as well as flapping wings generated conical LEVs that were stabilized by axial (radial) flow. Experiments conducted on insects in forward flight ${ }^{22}$ suggest that the inclination of the stroke plane to the direction of flight increases with increase in forward flight speed. This limited body of information suggests that one may assume that a limited degree of similarity exists between rotary and flapping wing vehicles. A wake surface for flapping wing vehicles in forward flight can be viewed to be somewhat similar to the concept of a prescribed wake that is sometimes used for a helicopter in forward flight. Specifically, one may assume that the wake is shed on a skewed cylindrical surface, denoted by $C S 2_{j}$, whose axis is inclined to the stroke plane at an angle $\chi_{c s 2}$ as depicted in Figures 3 and 4 respectively.

For a helicopter in forward flight, the tip path plane (TPP) is inclined at angle $\alpha_{R}$ to the direction of flight and the wake is skewed with respect to the axis of the rotor, as depicted in Figure 3. The skew angle, $\chi_{\mathrm{TPP}}$, is a function of the free stream velocity due to forward flight, and the induced velocity the represents the inflow normal to the rotor disk. According to Ref [23] (pp 453-458) an approximation that is used for prescribed skewed cylindrical wakes for helicopters in forward flight is given by Eq (1)

$$
\chi_{\mathrm{TPP}}=\tan ^{-1}\left(\frac{\lambda}{\mu}\right)
$$

where the inflow ratio and advance ratio are given by:

$$
\lambda=\frac{v_{i}}{R_{\text {span }} \Omega} \quad \text { and } \quad \mu=\frac{V_{\infty} \cos \alpha_{R}}{R_{\text {span }} \Omega}
$$

Note that the inflow ratio ${ }^{23}$ for helicopters in forward flight is estimated using momentum theory by Eq (3)

$$
\lambda=\mu \tan \alpha_{R}+\frac{C_{T}}{\sqrt{\mu^{2}+\lambda^{2}}}
$$

where $\alpha_{R}$ and $C_{T}$ are obtained from the trim state of a helicopter in steady level forward flight. It should be noted that the trim state of a flapping wing MAV cannot be clearly identified due to the highly unsteady operating conditions and lack of clearly defined control surfaces. Thus the similarities between a helicopter and a flapping wing MAV are speculative, and the current study represents a first attempt at incorporating forward flight into the formulation. 


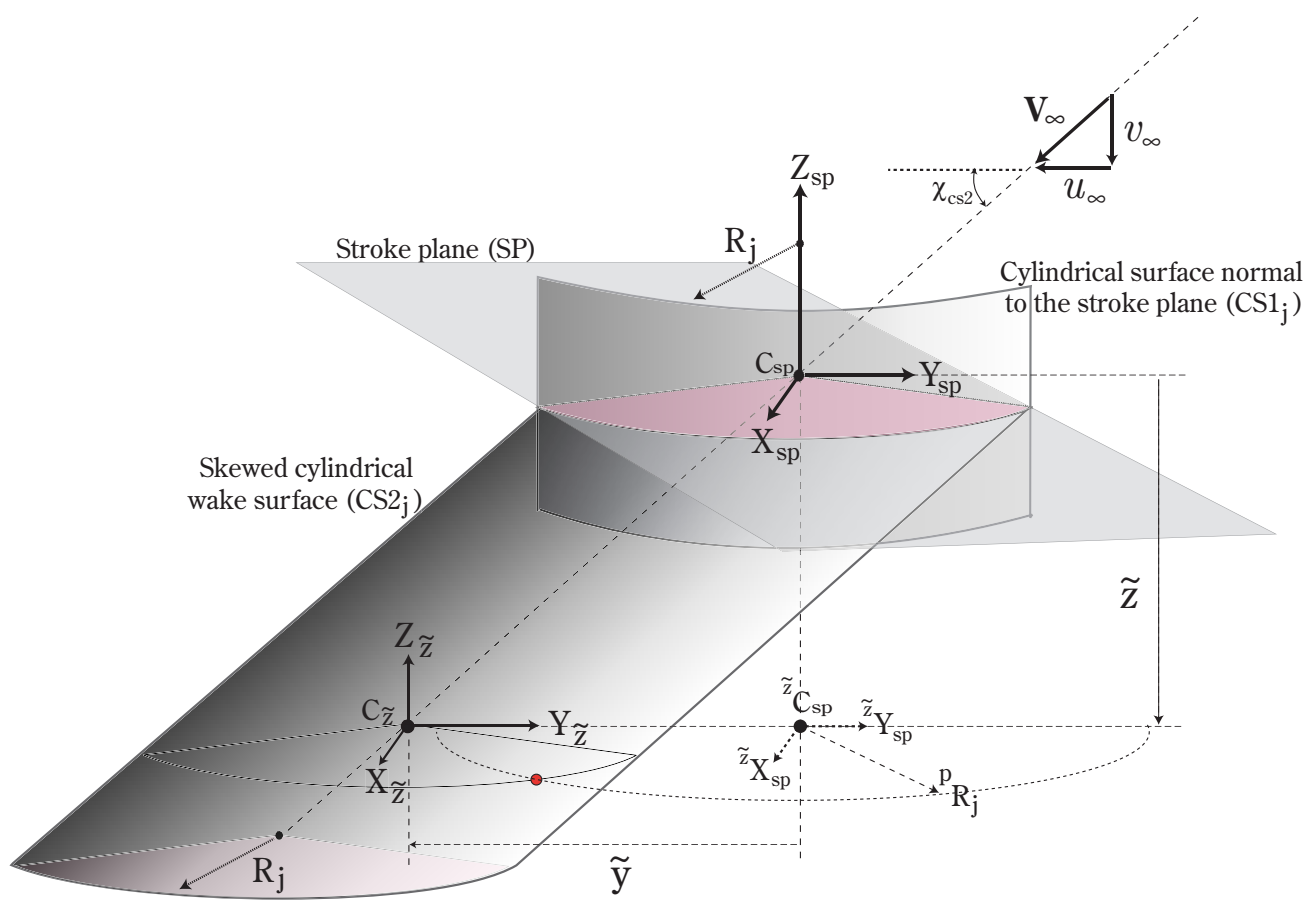

Figure 4. Surfaces used for each spanwise section. $X_{S P}-Y_{S P}$ defines the stroke plane (SP). Red dot indicates current location of a vortex particle moving on $C S 2_{j}$.

Thus, it is assumed that the skew angle of the wake surface for flapping wings is also given by a relation similar to Eq (1), provided that the advance ratio for a flapping wing MAV is given by:

$$
\mu=\frac{V_{\infty} \cos \beta_{S P}}{2 \pi f \beta_{0} R_{\text {span }}}
$$

where $\beta_{S P}$ is illustrated in Figure 3. A more complicated issue is the identification of the inflow velocity for a flapping wing MAV. Earlier attempts to compute induced velocity ${ }^{24,25}$ for flying insects were based on concepts of quasi-steady attached flow, momentum theory, and blade element theory. In Refs [24,25], the mean inflow velocity of a flapping wing in hover was obtained by performing a $2 \mathrm{D}$ analysis of a flapping wing and incorporating the mean lift coefficient into momentum theory. This approach ignored LEVs, wing rotation, and wing-wake interaction and therefore has questionable validity.

In the current study, the component of the free stream normal to the stroke plane is used as an approximation to the inflow velocity through the stroke plane. The stroke plane inclination angles observed for insects in forward flight are relatively large. Experiments on insects in hover and forward flight ${ }^{22}$ show that the values of $\beta_{S P}$, shown in Figure 3, are 20 degrees, 35 degrees, 45 degrees, and 60 degrees, for $\mu$ corresponding to $0,0.05,0.1$, and 0.15 respectively. Thus, the component of free stream velocity normal to the stroke plane can be substantial and it is assumed here that it provides an approximation to the inflow velocity. The validity of this approximation can be re-examined when additional experimental data and/or CFD based analyses of flapping wings in forward flight become available. The implications of this assumption are

$$
v_{i} \approx v_{\infty} \quad \text { such that } \quad \lambda=\frac{v_{\infty}}{2 \pi f \beta_{0} R_{\text {span }}}
$$

Substituting Eqs (4) and (5) into Eq (1), the skew angle for flapping wing vehicles is given by

$$
\chi_{c s 2}=\tan ^{-1}\left(\frac{v_{\infty}}{u_{\infty}}\right)=\beta_{S P}
$$

where $u_{\infty}$ and $v_{\infty}$ are shown in Figures 3 and 4 respectively. Note that Eq (6) is not valid for the case of hover, when both $u_{\infty} \rightarrow 0$ and $v_{\infty} \rightarrow 0$. Therefore, it is assumed that $\chi_{c s 2} \rightarrow \frac{\pi}{2}$ when $u_{\infty} \rightarrow 0$; this accounts for hover as well as bird-like flapping motion in which the direction of flight is normal to the stroke plane. 
For the general case, i.e. $u_{\infty} \neq 0$ and $v_{\infty} \neq 0, C S 1_{j}$ and $C S 2_{j}$ intersect on the stroke plane but do not overlap, as shown in Figure 4. Moreover, $C S 2_{j}$ intersects $C S 1$ 's associated with other wing sections. This implies that the interaction between the wing and the wake is three dimensional (3D) in spite of the simplifying assumptions used.

In the present analysis, an airfoil-wake surface that is a composite of $C S 1_{j}$ and $C S 2_{j}$, denoted by $C S 1_{j} \cup C S 2_{j}$ in Figure 5, is used to formulate the governing equations. It is divided into near and far wake regions to facilitate incorporation of the $3 \mathrm{D}$ wing-wake interaction in a computationally efficient manner. It is assumed that the $3 \mathrm{D}$ interaction is limited to the near wake region, wherein the near wake region is defined on $C S 1_{j}$ and has a height $z_{n w}$ as shown in Figure 5. The far wake region is defined on $C S 2_{j}$. An important step in modeling the 3D interaction involves projecting the positions and strengths of vortices from CS2's onto CS1's; this is described in subsequent sections. Also, note that the composite airfoil-wake surface is used to facilitate the conformal mapping and the subsequent calculation of airfoil bound and shed vorticity; however, this surface is not employed in the wake model.

\section{Conformal Mapping}

A crucial step in the hover formulation was the transformation of the airfoil-wake surface to a complex plane using a generalized Joukowski mapping. ${ }^{12}$ For the case of forward flight, the mapping function used in Ref [12] may be used without modification. Consequently, the airfoil on $C S 1_{j} \cup C S 2_{j}$ is transformed to a circle on a complex plane, where the complex plane is denoted by $\mathbb{C}_{j}$. The steps involved in the transformation are shown in Figure 5. Following the transformation, vorticity and circulation are computed on the circle in $\mathbb{C}_{j}$.

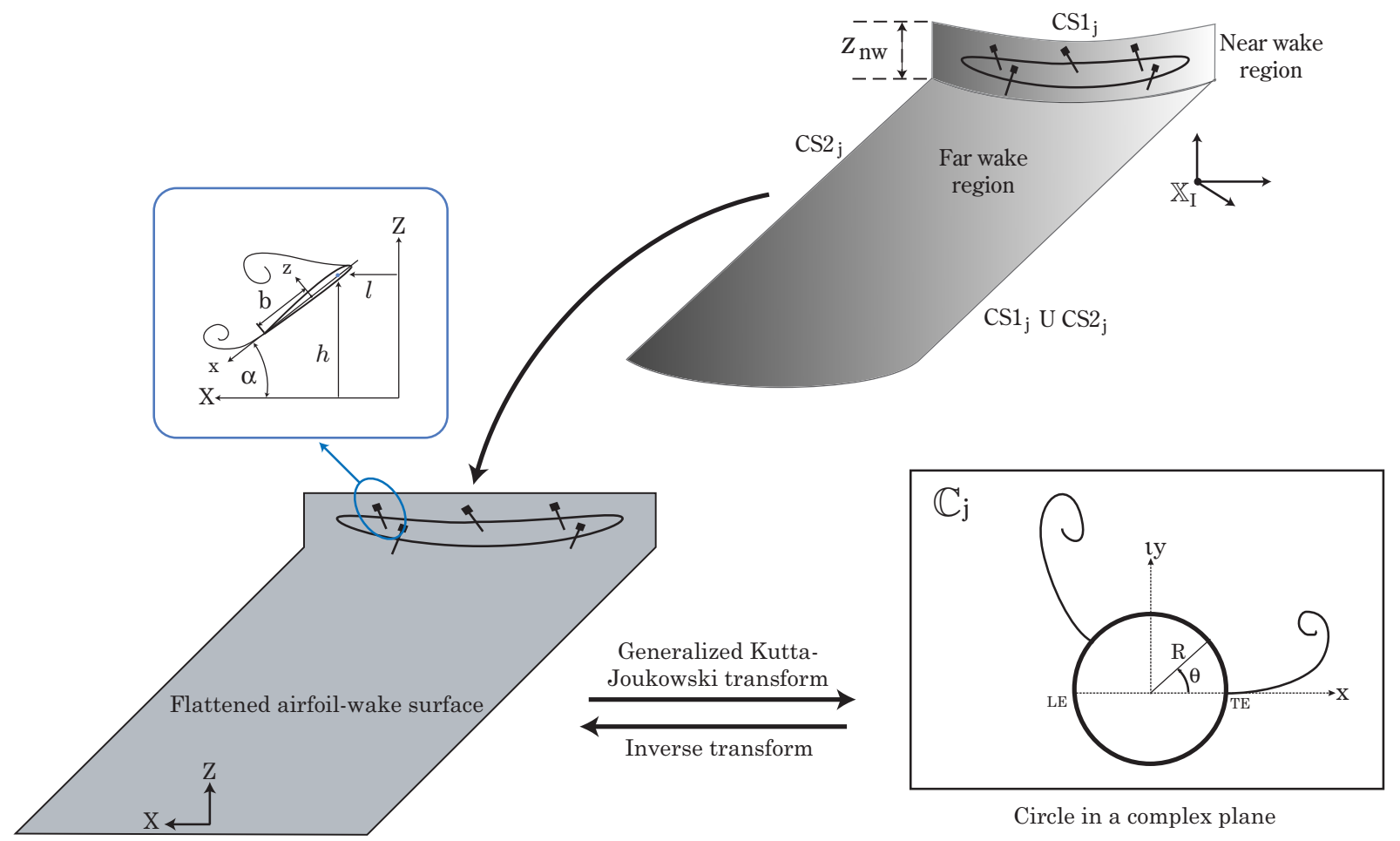

Figure 5. Transformation of the airfoil-wake to the circle in the complex plane.

\section{Quasi-steady Vorticity}

Quasi-steady vorticity, ${ }^{12}$ computed on the circle, is obtained as a sum of two components: (a) a free stream component that is computed from the instantaneous pitch angle and free stream velocity, and (b) an unsteady component that is computed from airfoil kinematics. 
Free stream component of vorticity

The free stream velocity vector, denoted by $\mathbf{V}_{\infty}$, is assumed to lie in the $Y_{S P}-Z_{S P}$ plane. It is resolved into components, $u_{\infty}$ along $Y_{S P}$ and $v_{\infty}$ along $Z_{S P}$ respectively, as shown in Figure 6(a).

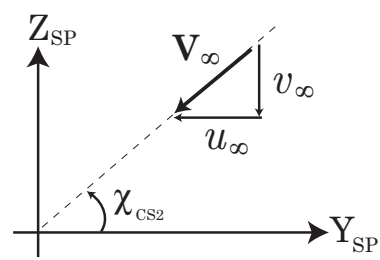

(a) Free stream velocity vector resolved normal and parallel to the stroke plane.

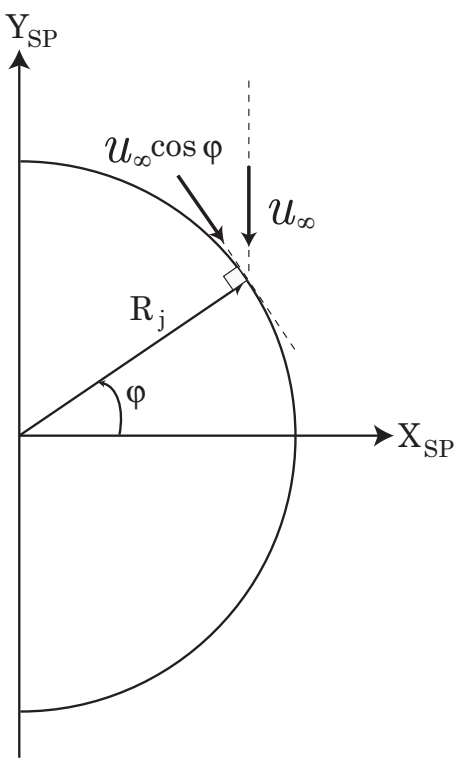

(b) Component of $u_{\infty}$ normal to the instantaneous position of the wing.

Figure 6. Components of free stream velocity vector.

The free stream velocity experienced by each wing section is assumed to be equal to $\left(\sqrt{\left(u_{\infty} \cos \varphi\right)^{2}+v_{\infty}^{2}}\right)$, where $\varphi$ denotes the instantaneous sweep angle of the feathering axis of the wing as shown in Figure 6(b) Subsequently, the vorticity and circulation due to the free stream velocity and pitch angle ${ }^{12}$ are given by

$$
\begin{aligned}
\left.\gamma_{0}(\theta, t)\right|_{f s} & =-2\left(\sqrt{\left(u_{\infty} \cos \varphi\right)^{2}+v_{\infty}^{2}}\right)[\sin (\theta-\alpha)+\sin \alpha] \\
\left.\Gamma_{0}(t)\right|_{f s} & =-4 \pi R\left(\sqrt{\left(u_{\infty} \cos \varphi\right)^{2}+v_{\infty}^{2}}\right) \sin \alpha
\end{aligned}
$$

\section{Unsteady component of vorticity}

The derivation and subsequent expression of the unsteady component is identical to the one presented in Ref [20]; The expression for the tangential velocity of the fluid on the surface of a flexible airfoil is given by

$$
\begin{aligned}
\left.v_{\theta}(\theta, t)\right|_{\text {us }}= & \frac{1}{R}\left[-A_{1} \cos \theta-\left(A_{2}+\frac{1}{2} A_{7}\right) \cos 2 \theta+A_{3} \sin \theta+\left(A_{4}-\frac{1}{2} A_{5}+\frac{1}{2} A_{6}\right) \sin 2 \theta\right. \\
& \left.-A_{8} \sin \theta \cos 2 \theta+A_{9} \sin \theta \sin 2 \theta-A_{10} \cos \theta \cos 2 \theta+A_{11} \cos \theta \sin 2 \theta\right] \\
& +\frac{\Delta v_{x}^{\text {flex }}}{R}[\tau(\sin \theta-\sin 2 \theta)+\sigma(\cos \theta-\cos 2 \theta)]+ \\
& +\frac{\Delta v_{y}^{\text {flex }}}{R}[-2 R \cos \theta+\tau(\cos \theta-\cos 2 \theta)-\sigma(\sin \theta-\sin 2 \theta)]
\end{aligned}
$$


where $A_{1}-A_{11}$ are given in Ref [12]. The vorticity on the circle is obtained as

$$
\left.\gamma_{0}(\theta, t)\right|_{u s}=\left.v_{\theta}(\theta, t)\right|_{u s}+\frac{\Gamma_{0}(t)}{2 \pi R}
$$

where

$$
\Gamma_{0}(t)=2 \pi\left[2 R(i \sin \alpha+\dot{h} \cos \alpha)+\dot{\alpha}\left(\frac{1}{2} \tau^{2}+\frac{1}{2} \sigma^{2}-2 R(R+a)\right)\right]
$$

\section{Determination of Shed Vorticity}

At each time step, the vorticity shed into the wake is determined by imposing a Kutta condition at the TE and a stagnation condition at the LE on the circle in the complex plane as follows:

At the TE:

$$
\Gamma_{0}=-\left[\oint_{l e v+w k} \mathcal{R}\left(\frac{Z_{v}+R}{Z_{v}-R}\right) d \Gamma_{v}\right]
$$

At the LE:

$$
\frac{1}{R}\left[A_{1}-\left(A_{2}+\frac{1}{2} A_{7}\right)+A_{10}\right]-2 U_{\infty} \sin \alpha=\frac{1}{2 \pi R}\left[\oint_{l e v+w k} \mathcal{R}\left(\frac{Z_{v}-R}{Z_{v}+R}\right) d \Gamma_{v}\right]
$$

where, $\mathcal{R}$ denotes real part.

Note that Eqs (11) and (12) are derived for vorticity on $\mathbb{C}_{j}$. For the case of hover, the airfoil as well as the wake vorticity are confined to $C S 1_{j}$; therefore, the strengths and locations of the vortices are obtained in a straightforward manner. For the case of forward flight, the vorticity shed by a section interacts with other sections, as mentioned earlier. Therefore, these effects have to incorporated in the wake integrals. In the present approach, the wake integrals that capture the effect of vorticity shed by other sections on $C S 1_{j} \cup C S 2_{j}$ are derived by projecting the vorticity that lies on $C S 2^{\prime}$ 's onto $C S 1_{j} \cup C S 2_{j}$.

\section{Projection of vorticity}

Based on the discussion on unsteady panel methods presented in Ref. [26], the irrotational inviscid 2D vortex particle used in Refs $[12,13,17,20]$ may be assumed to represent a 2D panel that has a single collocation point, as indicated in Figure 7. The width of the panel is equal to the width of the section from which it was shed, and length at the time of shedding is equal to the distance moved by the shedding edge (LE or TE) during a time step. This equivalence allows one to project wake vorticity on $C S 2_{j}$ onto $C S 1$ 's as follows:

Consider a vortex that is currently located on $C S 2_{j}$ at $z=\tilde{z}$, as indicated by the red dot in Figure 4 . Top view of the $z=\tilde{z}$ plane is shown in Figure 7. Let $\left(R_{j}, \phi_{j}\right)$ denote the radial and angular coordinates of the vortex in the $\left(X_{\tilde{z}}-Y_{\tilde{z}}\right)$ frame, as shown in Figure 7. The vortex represents a panel whose circulation vector $\mathbf{d} \boldsymbol{\Gamma}_{v}$ is given by

$$
\mathbf{d} \boldsymbol{\Gamma}_{v}=d \Gamma_{v} \hat{e}_{r}\left(\phi_{j}\right)
$$

where $\hat{e}_{r}\left(\phi_{j}\right)$, which denotes the unit vector in the radial direction as shown in Figure 7 , is given by

$$
\hat{e}_{r}\left(\phi_{j}\right)=\cos \phi_{j} \hat{e}_{X_{\tilde{z}}}+\sin \phi_{j} \hat{e}_{Y_{\tilde{z}}}
$$

Moreover, from Figure 17, note that

$$
\hat{e}_{X_{\tilde{z}}} \| \hat{e}_{\tilde{z}} X_{S P} \quad \text { and } \quad \hat{e}_{Y_{\tilde{z}}} \| \hat{e}_{\tilde{z}} Y_{S P}
$$

The radial and angular coordinates of the vortex in the $\left({ }^{\tilde{z}} X_{S P}-{ }^{\tilde{z}} Y_{S P}\right)$ frame, denoted by $\left(R_{k}, \phi_{k}\right)$ as shown in Figure 7, are given by

$$
R_{k}=\left[\left(R_{j} \cos \phi_{j}\right)^{2}+\left(R_{j} \sin \phi_{j}-\tilde{y}\right)^{2}\right]^{\frac{1}{2}}
$$

And

$$
\phi_{k}=\tan ^{-1}\left(\frac{R_{j} \cos \phi_{j}}{R_{j} \sin \phi_{j}-\tilde{y}}\right)
$$



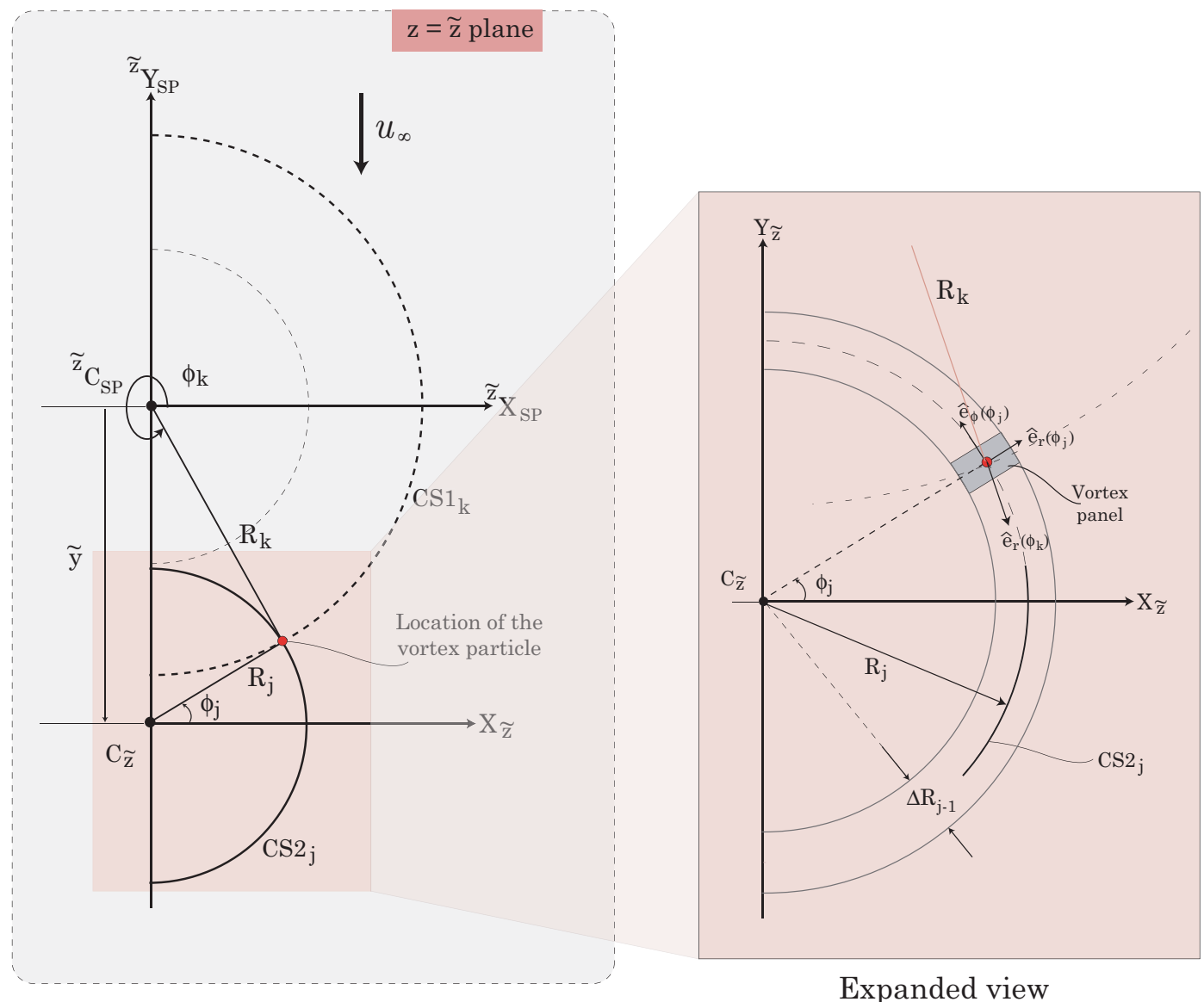

Figure 7. Top view - section of $C S 2_{j}$ located at $z=\tilde{z}$ in Figure 4 .

Where, the subscript ' $k$ ' denotes the $k^{\text {th }}$ spanwise station with respect to $C_{S P}$; for the general case $k \gtreqless j$. Furthermore, ${ }^{\tilde{z}} X_{S P},{ }^{\tilde{z}} Y_{S P}$, and ${ }^{\tilde{z}} C_{S P}$ denote the projections of $X_{S P}, Y_{S P}$, and $C_{S P}$ onto the $z=\tilde{z}$ plane.

Subsequently, vorticity is projected from $C S 2_{j}$ to $C S 1_{k}$; the strength of the projected vortex, denoted by ${ }^{p} \mathbf{d} \Gamma_{v}$, is given by

$$
{ }^{p} \mathbf{d} \boldsymbol{\Gamma}_{v}={ }^{p} d \Gamma_{v} \hat{e}_{r}\left(\phi_{k}\right) \quad \text { where } \quad{ }^{p} d \Gamma_{v}=d \Gamma_{v} \hat{e}_{r}\left(\phi_{j}\right) \cdot \hat{e}_{r}\left(\phi_{k}\right)
$$

Then from Eqs. (14), (15), and (18), one obtains

$$
{ }^{p} d \Gamma_{v}=d \Gamma_{v} \cos \left(\phi_{j}-\phi_{k}\right)
$$

Thus, a vortex that has strength $d \Gamma_{v}$ and located at $\left(R_{j}, \phi_{j}, \tilde{z}\right)$ on $C S 2_{j}$ corresponds to a projected vortex of strength ${ }^{p} d \Gamma_{v}$ located at $\left(R_{k}, \phi_{k}, \tilde{z}\right)$ on $C S 1_{k}$.

\section{Effect of a vortex on various airfoils}

In order to accurately model the 3D wing-wake interaction, it is necessary to quantify the effect of a shed vortex on various wing sections. In this context, it is important to note that the aerodynamic formulation is strictly $2 \mathrm{D}$ and does not support a mathematically consistent extension to $3 \mathrm{D}$. Inclusion of $3 \mathrm{D}$ effects in a mathematically consistent manner necessitates rederivation of the formulation that would also increase the computational expense significantly. Therefore, an ad hoc extension, which is obtained from aerodynamic analysis of fixed wings using 3D unsteady panel methods, ${ }^{26}$ is used. In this approach, an expression that approximates the effect of a projected vortex, located on $C S 1_{k}$, on an airfoil that is located on $C S 1_{j}, k \gtreqless j$, is derived as follows. 


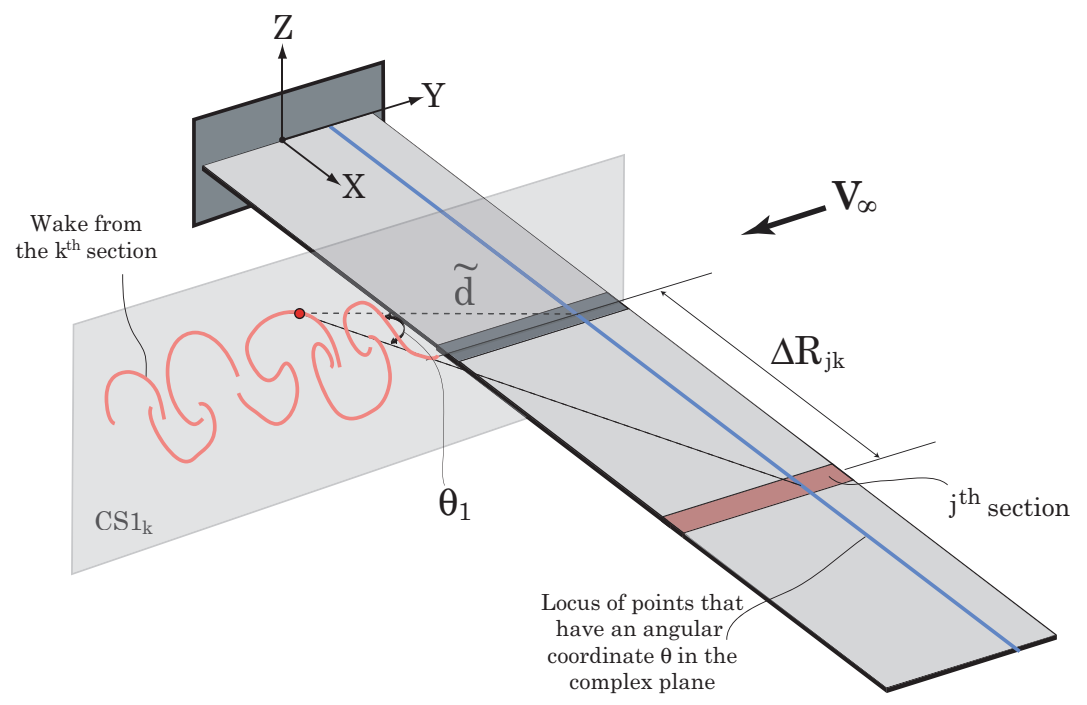

Figure 8. Effect of projected vorticity on adjacent sections.

Consider a uniform rectangular wing, as shown in Figure 8. All wing sections have the same geometric properties; consequently, the corresponding circles in $\mathbb{C}_{j}$ 's have the same radius $R$. Let $\Delta R_{j k}=\left|R_{k}-R_{j}\right|$ denote the distance between sections $C S 1_{j}$ and $C S 1_{k}$, as shown in Figure 8 . Let $\tilde{d}$ denote the distance between a vortex on $C S 1_{k}$ and a point on the airfoil section, also on $C S 1_{k}$, that has coordinates $(R, \theta)$ on $\mathbb{C}_{k}$. Then, the velocity induced by the vortex at this point is given by

$$
v_{\theta}=\frac{{ }^{p} d \Gamma_{v}}{2 \pi \tilde{d}}
$$

Now consider a point on the $j^{\text {th }}$ airfoil section that has coordinates $(R, \theta)$ on $\mathbb{C}_{j}$. The effect of the vortex at this point, obtained in the wing fixed $(X, Y, Z)$ coordinate system, is given by

$$
v_{\theta}=\frac{{ }^{p} d \Gamma_{v} \cos \theta_{1}}{2 \pi \sqrt{\tilde{d}^{2}+\left(\Delta R_{j k}\right)^{2}}}
$$

where $\theta_{1}$, shown in Figure 8, is given by

$$
\cos \theta_{1}=\frac{\tilde{d}}{\sqrt{\tilde{d}^{2}+\left(\Delta R_{j k}\right)^{2}}}
$$

From Eqs (21) and (22), one obtains

$$
v_{\theta}=\frac{{ }^{p} d \Gamma_{v} \cos \theta_{1}}{2 \pi \tilde{d}} \frac{\tilde{d}}{\sqrt{\tilde{d}^{2}+\left(\Delta R_{j k}\right)^{2}}}=\frac{\left({ }^{p} d \Gamma_{v} \cos ^{2} \theta_{1}\right)}{2 \pi \tilde{d}}
$$

Thus, the RHS of Eq (23) is obtained by replacing ${ }^{p} d \Gamma_{v}$ in Eq (20) with ${ }^{p} d \Gamma_{v} \cos ^{2} \theta_{1}$.

Equations $(20)$ - (23) are derived in physical space, denoted by $\mathbb{X}$; however, note that the shed vorticity is computed in the complex plane. Therefore, the validity of the relations in a space that is obtained via conformal transformation of the physical space need to be examined. In this context, consider the following argument: if an angle-preserving (conformal) mapping was used to transform the wing and its shed wake to a three dimensional solid and its shed wake, then, from the properties of conformal transformation it follows that quantities such as circulation and rotations (angles) are preserved. This implies that ${ }^{p} d \Gamma_{v}$ and $\theta_{1}$, and consequently ${ }^{p} d \Gamma_{v} \cos ^{2} \theta_{1}$, are preserved during the mapping. This implies that replacing ${ }^{p} d \Gamma_{v}$ with ${ }^{p} d \Gamma_{v} \cos ^{2} \theta_{1}$ in the transformed space is equivalent to the same replacement in physical space. 
The effect of a vortex that lies on $C S 1_{k}$ on other sections is obtained in three steps: (1) obtain the influence of the vortex at location $\theta$ on the circle on $C S 1_{k},(2)$ obtain $\tilde{d}$ and $\Delta R_{j k}$, as defined earlier, corresponding to the a point on $C S 1_{j}$ that has the same angular coordinate $\theta$, and (3) multiply the expressions obtained in step (1) by $\cos ^{2} \theta_{1}$ from Eq (22).

This derivation applies only to rectangular wings with uniform cross sections. For a different planform or varying geometric properties along the span, Eq (22) is no longer exact; however, Eqs (20) - (23) may still be used in an approximate sense.

\section{Modified Constraint Conditions}

Following the projection of vorticity, the Kutta condition, Eq (11), and the stagnation condition, Eq (12), are modified as follows:

Kutta condition:

$$
\Gamma_{0}=-\underbrace{\oint_{\text {lev+wk }} \mathcal{R}\left(\frac{Z_{v}+R}{Z_{v}-R}\right)^{p} d \Gamma_{v}}_{\text {Vortices on } C S 1_{j}}-\underbrace{\oint_{\text {lev+wk }} \mathcal{R}\left(\frac{Z_{v}+R}{Z_{v}-R}\right) d \Gamma_{v}}_{\text {Vortices on } C S 2_{j}}
$$

Stagnation condition:

$$
\begin{aligned}
\frac{1}{R}\left[A_{1}-\left(A_{2}+\frac{1}{2} A_{7}\right)+A_{10}\right]-2 U_{\infty} \sin \alpha & =\frac{1}{2 \pi R} \underbrace{\left[\oint_{l e v+w k} \mathcal{R}\left(\frac{Z_{v}-R}{Z_{v}+R}\right){ }^{p} d \Gamma_{v}\right]}_{\text {Vortices on } C S 1_{j}} \\
& +\frac{1}{2 \pi R} \underbrace{\left[\oint_{l e v+w k} \mathcal{R}\left(\frac{Z_{v}-R}{Z_{v}+R}\right) d \Gamma_{v}\right]}_{\text {Vortices on } C S 2_{j}}
\end{aligned}
$$

\section{Effect of shed vorticity}

The airfoil wake surface, shown in Figure 5, contains airfoil bound vorticity, projected vortices in the near wake region $\left(C S 1_{j}\right)$, and unprojected vortices in the far wake region $\left(C S 2_{j}\right)$. The effect of the shed vorticity at a point on the airfoil on $C S 1_{j}$ is given by the following integrals:

$$
\begin{aligned}
& \left(\left.\gamma_{1}(\theta, t)\right|_{w k+l e v}\right)_{j}=-\frac{\Gamma_{0}(t)}{2 \pi R} \\
& -\underbrace{\left\{\frac{1}{2 \pi R} \oint_{C} \mathcal{R}\left(\frac{Z_{v}+R e^{\imath \theta}}{Z_{v}-R e^{\imath \theta}}\right){ }^{p} d \Gamma_{v}+\frac{1}{2 \pi R} \oint_{C S 2_{j}} \mathcal{R}\left(\frac{Z_{v}+R e^{\imath \theta}}{Z_{v}-R e^{\imath \theta}}\right) d \Gamma_{v}\right\}}_{C S 1_{j}} \\
& -\sum_{\substack{k=1, k \neq j}}^{N_{\text {sections }}} \underbrace{\frac{1}{2 \pi R}\left(\oint_{C S 1_{k}} \mathcal{R}\left(\frac{Z_{v}+R e^{\imath \theta}}{Z_{v}-R e^{\imath \theta}}\right) p \Gamma_{v} \cos ^{2} \theta_{1}\right)}_{\text {Vortices on } C S 1_{k}, k \neq j}
\end{aligned}
$$

The second term in Eq (26) includes the effect of vorticity shed from other sections. Evaluation of this integral for the entire combination of vortices at all sections is computationally expensive; therefore, for a practical approximation, only a limited number of sections are considered using proximity argument. Thus, 
Eq (26) can be replaced by Eq (27).

$$
\begin{aligned}
\left(\left.\gamma_{1}(\theta, t)\right|_{w k+l e v}\right)_{j} & =-\frac{\Gamma_{0}(t)}{2 \pi R} \\
& -\underbrace{\left\{\frac{1}{2 \pi R} \oint_{C S 1_{j}} \mathcal{R}\left(\frac{Z_{v}+R e^{\imath \theta}}{Z_{v}-R e^{\imath \theta}}\right){ }^{p} d \Gamma_{v}+\frac{1}{2 \pi R} \oint_{C S 2_{j}} \mathcal{R}\left(\frac{Z_{v}+R e^{\imath \theta}}{Z_{v}-R e^{\imath \theta}}\right) d \Gamma_{v}\right\}}_{\text {Vortices on } C S 1_{j} \cup C S 2_{j}} \\
& -\sum_{\substack{k=j-M \\
k \neq j}}^{\frac{1}{2 \pi R}\left(\oint_{C S 1_{k}} \mathcal{R}\left(\frac{Z_{v}+R e^{\imath \theta}}{Z_{v}-R e^{\imath \theta}}\right){ }^{p} d \Gamma_{v} \cos ^{2} \theta_{1}\right)}
\end{aligned}
$$

\section{Calculation of the bound vorticity}

Following the derivation in Ref [12], the airfoil bound vorticity on the circle corresponding to the $j^{\text {th }}$ section is obtained as a combination of the wake free and wake induced components. The wake free component is obtained from the quasi-steady vorticity. The wake induced component is obtained as a sum of the contributions from the vortices on $C S 1_{j} \cup C S 2_{j}$ and $C S 1_{j \pm m}$, for $m=1,2, \ldots, M$.

$$
\left(\gamma_{b}\right)_{j}=\left(\left.\gamma_{0}\right|_{f s}\right)_{j}+\left(\left.\gamma_{0}\right|_{u s}\right)_{j}+\left(\left.\gamma_{1}\right|_{w k+l e v}\right)_{j \pm m}
$$

where, $m=0, \pm 1, \pm 2, \ldots, \pm M$, and $\left.\gamma_{0}\right|_{f s},\left.\gamma_{0}\right|_{u s}$, and $\left.\gamma_{1}\right|_{w k+l e v}$ are given in Eqs (7), (9), and (27) respectively.

\section{Wake evolution}

We assume that the shed wake, which is confined to $C S 2_{j}$, is not affected by vorticity associated with other sections. Consequently, the evolution of the wake, obtained from the Rott-Birkhoff equation, is determined on $C S 2_{j}$. The expression for velocity of each vortex has contributions from airfoil bound vorticity, which is projected from $C S 1_{j}$ to $C S 2_{j}$, and shed vortices on $C S 2_{j}$. The final expression for velocity and subsequent numerical implementation of the wake model follow from the description provided in Refs $[12,13,17,20]$.

\section{The Aeroelastic Model}

The equations of motion representing the aeroelastic response problem are obtained from an updated Lagrangian (UL) approach. ${ }^{27,28}$ An approximate solution is obtained by referring all the quantities (stress, strain and displacements) of the deformed configuration to the equilibrium configuration obtained in the previous time step, and linearizing the resulting equations of motion (EOM). Implementation of the UL formulation in MARC 27,28 is illustrated by Figure 9 and was summarized in Ref. [17]. The implementation of the aeroelastic model is shown in the block diagram given in Figure 9, At each time step, rigid body motion is prescribed as displacements at specified nodes. The aerodynamic loads, computed based on the wing motion at the beginning of each time step, are applied to the structure via FORCEM, a user defined subroutine in MARC. This subroutine is called from the main program for each step of the Newton-Raphson iteration within a time step to ensure convergence of the structural displacements for the applied loads. Finally, the vortices shed into the wake are convected at the end of the time step.

\section{Results and Discussion}

The results presented in this section consist of different groups: (1) structural dynamic comparisons with experimental data, (2) rigid and isotropic flapping wings in hover, (3) anisotropic flapping wings in hover, and (4) preliminary results on rigid flapping wings in forward flight. All the results are based on a Zimmerman planform, shown in Figures 2 and 11, that have an aspect ratio of 7.65, $c_{r}=25 \mathrm{~mm}, R_{\text {span }}=75$ mm respectively. ${ }^{29}$ The finite element models of the wings, shown in Figure 2, are obtained using 1263 


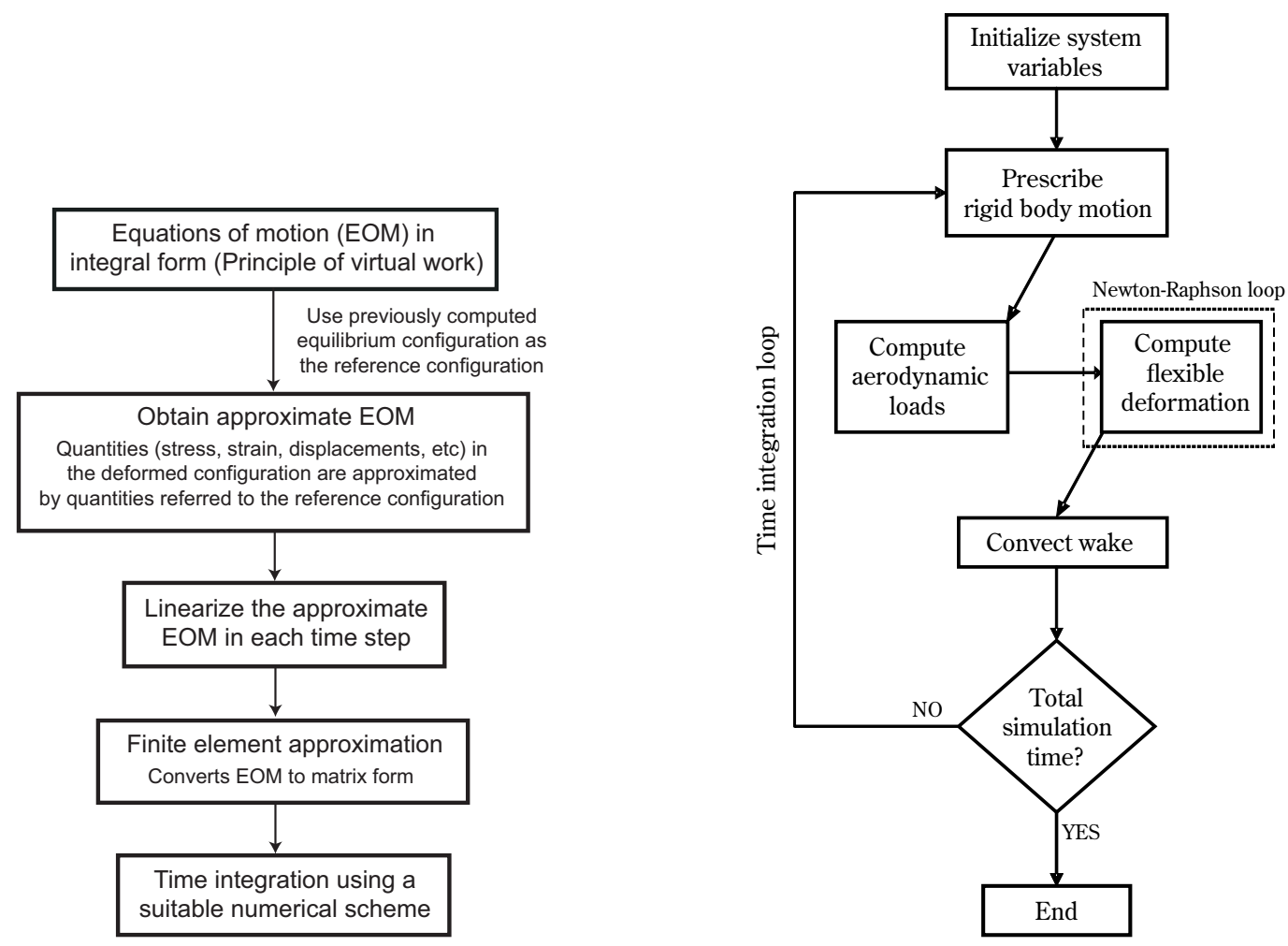

Figure 9. Formulation of the aeroelastic equations (left) and implementation of the aeroelastic model (right) in MARC

shell elements (Element type 75 in MARC) that are capable of modeling large rigid body motion as well as moderate-to-large flexible deformation.

The unsteady aerodynamic loads calculated using the approximate aerodynamic model were computed for the following set of parameters: $N_{\text {sections }}=59, N_{\theta}=101, r_{c}=0.1 \times$ chord, and $n_{w k s u b i t}=4$ respectively. The numerical experiments were conducted in air $\left(\rho_{\infty}=1.209 \mathrm{~kg} / \mathrm{m}^{3}\right)$ and leading edge separation was assumed for all cases. A previous study ${ }^{20}$ noted that the interaction of the airfoil with previously shed vortices generated large amplitude numerical oscillations in the aerodynamic loads that had to be eliminated before the unsteady loads could be applied on the structure. In the current study, a pressure based filter that is described by $\mathrm{Eq}(29)$ was found to limit the magnitude of numerical noise without introducing significant errors in the calculation of pressure.

$$
p_{\text {applied }}=\operatorname{maximum}\left\{p_{\text {calculated }}, p_{\text {limit }}\right\}
$$

where,

$$
p_{\text {limit }}=\frac{1}{2} \rho_{\infty} U_{\text {tip }}^{2} \quad \text { where } \quad U_{\text {tip }}=2 \pi f \beta_{0} R_{\text {span }}
$$

Subsequently, the load signals obtained from the approximate model are smoothed using digital filters available in MATLAB, version 8.0, for the purpose of presentation in this paper. The filters are based on Chebyshev functions and are implemented in MATLAB using the filtfilt command. A sample comparison of the original and smoothed signals is shown in Figure 10. Note that all figures associated with the results section are presented at the end of the document.

\section{A. Structural Dynamic Comparisons}

The computed and experimentally determined ${ }^{29}$ mode shapes and frequencies for anisotropic Zimmerman wings are compared so as to validate the structural dynamic models. The anisotropic wings, shown in Figure 12, are built from an unstressed CAPRAN film (membrane) that is supported by a carbon fiber based sparbatten skeleton. Using the notation used in Ref [29], the wings are labeled as LiBj where i and $\mathrm{j}$ denote the 
number of prepreg layers used in the construction of the LE spar and the battens, shown in Figure 11. The nominal elastic properties of the materials used were provided by the respective manufacturers.

The natural frequencies of the wings were identified using a Laser Doppler Vibrometer (LDV) that uses the phase shift between the incident and reflected light to obtain a frequency response spectrum of the structure. ${ }^{29}$ The experiments involve visual tracking of the wing surface using high speed cameras to obtain the deformation pattern. The translucent CAPRAN film, shown in Figure 12 (right), is not conducive to visual tracking. Therefore, a dense speckle pattern was generated by spraying black acrylic paint, shown in Figure 12 (left), to facilitate tracking of the wing. The CAPRAN film is very thin, lightweight, and flexible, and the change in geometric and material properties of the film due to the paint speckle that is deposited on the film had to be determined experimentally. Tests to measure thickness and Young's modulus of the films were done at the University of Michigan. Subsequently, these properties were used to generate the structural dynamic and aeroelastic results.

\section{Tensile Tests on the CAPRAN Membrane}

The Young's moduli of the films were measured by tensile tests using specimens shown in Figure 13. Tensile test samples are manufactured to a total length of $25 \pm 1 \mathrm{~mm}$, a gauge length of $13 \pm 2 \mathrm{~mm}$ and a height of $2.5 \pm 0.5 \mathrm{~mm}$. The tests were carried out under displacement control loading at a nominal rate of 0.018 $\mathrm{mm} / \mathrm{s}$. The small scale tensile tester is shown in Figure 14. Strains are determined using optical images taken with a high resolution camera of a speckle pattern, distributed on the surface of the film using acrylic paint applied with an airbrush or a spray can. Unpainted films could not be tracked; therefore, a light speckle pattern, expected to have an insignificant effect on the material properties of the film, was deposited on the unpainted films. Specimens of the densely and lightly painted films, labeled as Heavy dots and Light dots are shown in Figure 15. Thicknesses of the samples are measured with the aid of a Phillips XL30 Environmental Scanning Electron Microscope (SEM).

The SEM measurements indicated that there was little or no change in thickness of the film due to the paint; an average value of 15 microns is therefore used in calculations. The thicknesses are listed in Table 1. a sample image from the SEM is shown in Figure 16. The characteristic stress-strain curves obtained after the post-processing of the data are shown in Figures 17(a) and 17(b). The results indicate that the elastic moduli of the painted (heavy dots) and lightly painted (light dots) films are approximately the same, where an averaged value of $\mathrm{E}=2.74 \mathrm{GPa}$ is used in calculations. However, static weight measurements show that the paint increases the weight, and the density, of the films by approximately $16 \%$. The densities and Young's moduli of the painted and unpainted films are given in Table 1

\begin{tabular}{|c|c|c|}
\hline & Unpainted Film & Painted film \\
\hline Density, $\mathrm{kg} / \mathrm{m}^{3}$ & 1186.0 & 1383.7 \\
\hline Young's modulus, GPa & $2.72 \pm 0.16$ & $2.76 \pm 0.21$ \\
\hline Thickness, $\left(\times 10^{-6} \mathrm{~m}\right)$ & $15.17 \pm 0.90$ & $14.96 \pm 0.57$ \\
\hline
\end{tabular}

Table 1. Density and Young's moduli of the painted and unpainted films.

\section{Comparison of Mode Shapes and Frequencies}

The experimentally determined and computed mode shapes and frequencies for anisotropic Zimmerman wings are shown in Figure 18 and Tables 4 and 5 respectively.

Note that the experiments ${ }^{29}$ were conducted in air; this implies that the mode shapes and frequencies that are identified correspond to aeroelastic modes that include added mass effects ${ }^{7}$ as well as coupling effects between bending and torsion. In an aeroelastic system, a useful indicator of the relative importance of the inertial to aerodynamic forces is the mass ratio $^{7}$ that is described by Eq (31).

$$
\text { Mass ratio }=\frac{\rho t_{h} c_{r}}{0.25 \pi \rho_{\infty} c_{r}^{2}}
$$

where, the thickness of the composite and the membrane are given in Table 3 . A lower bound of the mass ratio for the anisotropic wings is obtained by considering a representative cross section that is composed entirely of the membrane material, illustrated in Figure 19 (left). The mass ratio can also be computed by 
considering a more realistic cross section as shown in Figure 19 (right). The values of the mass ratios are given in Table 2. The calculations indicate that the added mass effects are most likely to be significant for modes that have a predominant contribution from the membrane, and these will be relatively insignificant for modes that have a predominant contribution from the composite skeleton. An examination of the experimentally determined mode shapes shows that the first mode has a predominant contribution from the skeleton; this indicates that the first measured frequency is a reasonable approximation to the first natural frequency.

\begin{tabular}{|c|c|}
\hline & Mass ratio \\
\hline Cross-section composed of membrane material & 0.88 (lower bound) \\
\hline Realistic cross-section & 235 (L1B1) \\
\hline
\end{tabular}

Table 2. Mass ratios corresponding to sample cross-section of the wing

The finite element models use the nominal values for the wing geometry, material and elastic properties. However, the elastic properties of the carbon fiber prepreg are modified by adjusting the recommended properties so as to obtain a reasonable overall correlation with experimentally obtained frequencies and mode shapes. The recommended and adjusted elastic properties are given in Table 3 .

\begin{tabular}{|c|c|c|}
\hline & Recommended values & $\begin{array}{c}\text { Adjusted values used in the } \\
\text { structural dynamic model }\end{array}$ \\
\hline Carbon fiber prepreg & $E_{11}=233 \mathrm{GPa}$ & $E_{11}=233 \mathrm{GPa}$ \\
& $E_{22}=23.1 \mathrm{GPa}$ & $E_{22}=23.1 \mathrm{GPa}$ \\
(Properties of one layer) & $E_{12}=3 \mathrm{GPa}$ & $E_{12}=\mathbf{1 0 . 5} \mathrm{GPa}(\mathrm{L} 1 \mathrm{~B} 1, \mathrm{~L} 1 \mathrm{~B} 2)$ \\
& $E_{12}=\mathbf{1 5 . 5} \mathrm{GPa}$ (all other configs) & $\nu_{12}=0.05$ \\
& $\nu_{12}=0.05$ & $\rho=1740 \mathrm{~kg} / \mathrm{m}^{3}$ \\
& $\rho=1740 \mathrm{~kg} / \mathrm{m}^{3}$ & Thickness $=0.1 \mathrm{~mm}$ \\
\hline Capran membrane & Thickness $=0.1 \mathrm{~mm}$ & $E=2.76 \mathrm{GPa}$ \\
(From experiments) & $E=2.5-3.5 \mathrm{GPa}$ & $\nu_{12}=0.489($ Incompressible) \\
& $\rho=1160 \mathrm{~kg} / \mathrm{m}^{3}$ & $\rho=\mathbf{1 3 8 4} \mathrm{kg} / \mathrm{m}^{3}$ \\
& Thickness $=12-20 \mathrm{microns}$ & Thickness $=15 \mathrm{microns}$ \\
\hline
\end{tabular}

Table 3. Material properties of the composite and membrane

\begin{tabular}{|c|c|c|c|}
\hline Wing label & & Mode 1 & Mode 2 \\
\hline & Experiment & 23 & 50 \\
L1B1 & FE model & 21.5 & 49 \\
\hline & Experiment & 22 & 45 \\
L1B2 & FE model & 19.5 & 46.2 \\
\hline & Experiment & 42 & 84 \\
L2B1 & FE model & 47 & 88 \\
\hline & Experiment & 41 & $\mathbf{5 2}$ \\
L2B2 & FE model & 44 & 86.1 \\
\hline & Experiment & 59 & 104 \\
L3B1 & FE model & 65 & 107 \\
\hline & Experiment & 67 & $\mathrm{n} / \mathrm{a}$ \\
L3B2 & FE model & 64 & 101 \\
\hline
\end{tabular}

Table 4. Comparison of frequencies, in $\mathrm{Hz}$, of various wing configurations

A comparison of the frequencies, shown in Table 4, indicates that there is reasonable agreement (approx $20 \%$ error) between the computed and measured values. The computations yield closely spaced modes, listed 
in Table 5, that contain a predominant contribution from the membrane. These modes were not identified in the experiments. However, the frequency spectrum obtained from the experiments ${ }^{29}$ show several smaller peaks that were ignored. It is conceivable that these correspond to the closely spaced modes. The cases that show significantly higher error are identified in bold. The experiments indicate a decrease in the natural frequency for the second and third modes from $84 \mathrm{~Hz}$ and $126 \mathrm{~Hz}$ for L2B1 to $52 \mathrm{~Hz}$ and $84 \mathrm{~Hz}$ for L2B2. This decrease appears to be questionable based on the trends observed in the other configurations. This discrepancy may be due to the manner in which the modes were identified from the frequency response spectrum. $^{29}$

\begin{tabular}{|c|c|c|c|c|c|c|}
\hline Wing label & Mode 1 & Mode 2 & Mode 3 & Mode 4 & Mode 5 & Mode 6 \\
\hline L1B1 & $\mathbf{2 1 . 5}$ & $\mathbf{4 9}$ & 73.8 & 77.4 & $\mathbf{1 0 6 . 5}$ & 111.5 \\
\hline L1B2 & $\mathbf{1 9 . 5}$ & $\mathbf{4 6 . 2}$ & 74.2 & 78.5 & $\mathbf{1 0 0 . 5}$ & 107.5 \\
\hline L2B2 & $\mathbf{4 7}$ & 72 & 76.5 & $\mathbf{8 8}$ & 109 & $\mathbf{1 1 8 . 8}$ \\
\hline L2B2 & $\mathbf{4 4}$ & 74 & 78.7 & $\mathbf{8 6 . 1}$ & 109 & $\mathbf{1 1 8 . 5}$ \\
\hline L3B2 & $\mathbf{6 5}$ & 75.5 & 76.8 & $\mathbf{1 0 7}$ & 109 & 120 \\
\hline L3B2 & $\mathbf{6 4}$ & 78 & 79 & $\mathbf{1 0 1}$ & 109 & 120 \\
\hline
\end{tabular}

Table 5. Computed frequencies, in $\mathrm{Hz}$, of various wing configurations. Frequencies used for comparison with experiment are identified in bold.

A qualitative comparison of the mode shapes obtained for L1B1 and L1B2 is shown in Figure 18. These results indicate that the FE model shows reasonable agreement with the experimental results for the cases considered.

The numerical simulations indicate that the shear moduli of the materials used has a significant impact on the placement and spacing of frequencies. Therefore, additional studies that examine the sensitivity of the measured frequencies, and consequently the thrust generated, due to variations in the material properties may be necessary.

\section{B. Rigid and Isotropic Flapping Wings in Hover}

The lift and thrust generated by rigid and isotropic wings undergoing prescribed flapping motion are shown in Figure 20. Note that the lift and thrust are the components of the aerodynamic force resolved parallel to and normal to the stroke plane respectively. The flapping motion, which corresponds to rotation about $Z_{S P}$ shown in Figure 4, is described by Eq (32)

$$
\beta(t)=\beta_{0} \sin (2 \pi f t)
$$

where $\beta_{0}=5 \operatorname{deg}$ and $f=10 \mathrm{~Hz}$. The wing thickness, density, and Poisson's ratio were $0.4 \mathrm{~mm}, 2700 \mathrm{~kg} / \mathrm{m}^{3}$, and 0.3 respectively. The number of time steps per flapping cycle used to discretize the motion for each case are listed in Table 6. The CFD based results, which are presented in Ref [30], were computed using the aeroelastic model that is described in Refs $[18,19]$. The lift and thrust were non-dimensionalized as described by Eq (33).

$$
C_{L}=\frac{L}{\frac{1}{2} \rho_{\infty} U_{\text {tip }}^{2} R_{\text {span }} c_{\text {root }}} \quad \text { and } \quad C_{T}=\frac{T}{\frac{1}{2} \rho_{\infty} U_{\text {tip }}^{2} R_{\text {span }} c_{\text {root }}} \quad \text { where } \quad U_{\text {tip }}=4 \pi \beta_{0} f
$$

The results, shown in Figure 20, indicate that the approximate aeroelastic model compares well with the CFD based model for the cases considered. Note that a periodic or steady state solution was not observed for the case of $\mathrm{E}=0.1 \mathrm{GPa}$; therefore, an accurate assessment of the differences between the CFD and approximate result is difficult.

\begin{tabular}{|c|c|c|c|c|}
\hline E, GPa & Rigid & 70 & 10 & 0.1 \\
\hline Time steps per cycle & 200 & 200 & 250 & 300 \\
\hline
\end{tabular}

Table 6. Number of time steps per cycle used for the isotropic wing cases 


\section{Anisotropic Flapping Wings in Hover}

The computed and experimentally measured magnitudes of thrust generated by anisotropic wings are compared. The results, which are obtained for a flapping amplitude of $35^{\circ}$ for a range of flapping frequencies, are shown in Figures 21, 22, and 23 respectively. A relative comparison of thrust generated by all configurations is shown in Figure 24. The experimental results were obtained from Ref [29] and computations are based on the approximate aeroelastic model. Note that the experiments were conducted using two wings undergoing symmetric flapping motion, whereas the computations were performed by considering a single wing; therefore, the thrust obtained from the simulations was multiplied by a factor of two to facilitate comparison with the experimental result. The number of time steps used per flapping cycle for each case are summarized in Table 7. Furthermore, the circulation limit ${ }^{20}$ was fixed at 2.0. For all the cases considered, simulations were carried out for a total of 6 flapping cycles, and an approximate steady state in forces was reached after about 2 cycles.

\begin{tabular}{|c|c|c|c|c|c|c|c|c|}
\hline & $5 \mathrm{~Hz}$ & $10 \mathrm{~Hz}$ & $15 \mathrm{~Hz}$ & $20 \mathrm{~Hz}$ & $25 \mathrm{~Hz}$ & $30 \mathrm{~Hz}$ & $35 \mathrm{~Hz}$ & $40 \mathrm{~Hz}$ \\
\hline L1B1, L1B2 & 200 & 200 & 250 & 400 & 400 & 500 & 600 & 600 \\
\hline L2B1, L2B2 & 200 & 200 & 200 & 250 & 300 & 350 & 400 & 400 \\
\hline L3B1, L3B2 & 200 & 200 & 200 & 250 & 300 & 350 & 400 & 400 \\
\hline
\end{tabular}

Table 7. Number of time steps per cycle used for various wing configurations for the range of flapping frequencies considered.

It is evident from the results that the approximate aeroelastic model under-predicts the thrust for all cases considered. However, some of the trends are captured in an accurate manner: specifically, the peak in thrust observed for L1B1 for flapping frequency in the vicinity of its first natural frequency is important; see Figure 21. Furthermore, the computations indicate that the thrust generated reduces as the number of prepreg layers in the battens is increased. An explanation of this behavior is due to the fact that reinforcing the battens increases the torsional stiffness of the wing thereby reducing the twist angle of the wing. Therefore, the total force generated by the wing and its component normal to the stroke plane, i.e. thrust, are both reduced. This trend is not evident from the experimental results.

\section{Preliminary Forward Flight Results}

The effect of forward flight on the lift production is examined by considering rigid Zimmerman wings undergoing prescribed flap motion for a range of free stream velocities. Preliminary results were obtained for small amplitude flap motion for the case of $z_{n w}=0$ wherein the effect of vortices shed by other sections is not considered, i.e. the 3D effects due to forward flight are ignored; however, the movement of the vortices on the skewed wake surface is considered by using the time dependent projected strengths of the vortices in the calculation of shed and bound vorticity.

Results were obtained for $\chi_{c s 2}=20$ degrees, $\beta_{0}=5$ degrees, $f=10 \mathrm{~Hz}$, and $\mu=0.0,0.05,0.1,0.15,0.2$, respectively, where $\mu$ is defined in Eq (4) and the flapping motion is described by Eq (32). For the cases considered, the wing sections are normal to the stroke plane due to the absence of wing feathering or twisting; consequently, the thrust produced, which is the component of the aerodynamic force parallel to the wing chord, was several orders of magnitude smaller than the lift. Therefore, the thrust is not included for comparison purposes. Figure 25 shows the lift coefficients generated by the rigid wing for case of hover and forward flight. The results show an increase in lift due to forward flight. Note that the peaks in lift that correspond to the advancing part of the stroke are greater than the peaks that correspond to the retreating side. This corresponds to the effect of a time varying free stream that arises due to the flapping motion of the wing and the forward flight speed.

\section{Concluding Remarks}

The approximate aerodynamic model for flapping wings, originally developed for hover, is extended to forward flight. Results for structural dynamic studies on anisotropic wings, aeroelastic studies on isotropic and anisotropic wings, are also presented in the paper. 
1. Forces computed using the approximate aeroelastic model were compared with those obtained from a CFD-based aeroelastic model and experiments for several Zimmerman wing configurations for the case of hover. The comparisons indicate that the approximate model shows good agreement with CFD for the cases considered. Comparisons with the results obtained from experimental studies on anisotropic wings indicate that the approximate model captures some of the important trends accurately, but under predicts magnitude of the thrust generated. Additional tests are needed before an accurate assessment of the error is possible.

2. Preliminary results obtained for rigid flapping wings in forward flight indicated that forward flight increases lift generation for prescribed wing kinematics. The results also show that the lift generated during the advancing half of the cycle is larger compared to the force generated during the retreating half of the stroke. Subsequent studies will focus on comparisons with available results for flapping wings in forward flight, as well as examination of the effect of wing flexibility.

\section{Acknowledgments}

This work was supported by the Air Force Office of Scientific Research's (AFOSR) MURI with Dr. Douglas R. Smith as Program Director. The first author would like to thank Pin Wu, Robert Love, and Prof. Ifju for providing the anisotropic wings, the CAPRAN membrane, and composite sheets, for testing, and Devesh Kumar and Adam Olshove for help with the experiments on the CAPRAN membrane.

\section{References}

\footnotetext{
${ }^{1}$ Mueller, T. J., Fixed and Flapping Wing Aerodynamics for Micro Air Vehicle Applications, Vol. 195, Progress in Aeronautics and Astronautics, published by AIAA, 2001.

${ }^{2}$ Platzer, M. E. and Jones, K., "Flapping Wing Aerodynamics - Progress and Challenges," $44^{\text {th }}$ AIAA Aerospace Sciences Meeting and Exhibit, Reno, Nevada, January 2006, AIAA Paper Number 2006-500.

${ }^{3}$ Sane, S. P., "The Aerodynamics of Insect Flight," The Journal of Experimental Biology, Vol. 206, 2003, pp. 4191 - 4208.

${ }^{4}$ Ansari, S. A., Zbikowski, R., and Knowles, K., "Aerodynamic Modelling of Insect-like Flapping Flight for Micro Air Vehicles," Progress in Aerospace Sciences, Vol. 42, 2006, pp. 129 - 172.

${ }^{5}$ Shyy, W., Lian, Y., Tang, J., Liu, H., Trizila, P., Stanford, B., Bernal, L., Cesnik, C., Friedmann, P., and Ifju, P., "Computational Aerodynamics of Low Reynolds Number Plunging, Pitching, ad Flexible Wings for MAV Applications," 48 ${ }^{t h}$ AIAA Aerospace Sciences Meeting and Exhibit, Reno, Nevada, January 2008, AIAA Paper No. 2008-523.

${ }^{6}$ Shyy, W., Lian, Y., Tang, J., Viieru, D., and Liu, H., Aerodynamics of Low Reynolds Number Flyers, Cambridge University Press, 2008.

${ }^{7}$ Bisplinghoff, R. L., Ashley, H., and Halfman, R. L., Aeroelasticity, Addison Wesley Co., 1955.

${ }^{8}$ Singh, B. and Chopra, I., "An Aeroelastic Analysis for the Design of Insect-Based Flapping Wings," $48^{t h}$ AIAA/ASME/ASCE/AHS/ASC Structures, Structural Dynamics, and Materials Conference, Honolulu, Hawaii, April 2007, AIAA Paper Number 2007-1757.

${ }^{9}$ Polhamus, E. C., "A Concept of the Vortex Lift of Sharp Edge Delta Wings Based on a Leading-Edge Suction Analogy," Nasa tn d-3767, 1966.

${ }^{10} \mathrm{Katz}$, J., "A Discrete Vortex Method for the Non-steady Separated Flow over an Airfoil," Journal of Fluid Mechanics, Vol. 102, 1981, pp. 315-328.

${ }^{11}$ Shukla, R. K. and Eldredge, J. D., "An Inviscid Model for Vortex Shedding from a Deforming Body," Theoretical and Computational Fluid Dynamics, Vol. 21, 2007, pp. 343-368.

${ }^{12}$ Ansari, S. A., Zbikowski, R., and Knowles, K., "Non-linear Unsteady Aerodynamic Model for Insect-like Flapping Wing in the Hover. Part 1: Methodology and Analysis," Proceedings of the I MECH E Part G Journal of Aerospace Engineering, Vol. 220, No. 2, 2006, pp. 61-83.

${ }^{13}$ Ansari, S. A., Zbikowski, R., and Knowles, K., "Non-linear Unsteady Aerodynamic Model for Insect-like Flapping Wing in the Hover. Part 2: Implementation and Validation," Proceedings of the I MECH E Part G Journal of Aerospace Engineering, Vol. 220, No. 2, 2006, pp. 169-186.

${ }^{14}$ Combes, S. A. and Daniel, T. L., "Into Thin Air: Contributions of Aerodynamic and Inertial-Elastic Forces to Wing Bending in the Hawkmoth Manduca Sexta," The Journal of Experimental Biology, Vol. 206, 2003, pp. 2999 - 3006.

${ }^{15}$ Daniel, T. L. and Combes, S. A., "Flexible Wings and Fins: Bending by Inertial or Fluid-Dynamic Forces," Integrative and Comparative Biology, Vol. 42, 2002, pp. $1044-1049$.

${ }^{16}$ Hamamoto, M., Ohta, Y., Hara, K., and Hisada, T., "Application of Fluid-Structure Interaction Analysis to Flapping Flight of Insects with Deformable Wings," Advanced Robotics, Vol. 21, No. 1-2, 2007, pp. 1-21.

${ }^{17}$ Gogulapati, A., Friedmann, P., and Shyy, W., "Nonlinear Aeroelastic Effects in Flapping Wing Micro Air Vehicles," 49th AIAA/ASME/ASCE/AHS/ASC Structures, Structural Dynamics, and Materials Conference, No. AIAA Paper Number 2008-1817, Schaumburg, IL, April 7 - 102008.

${ }^{18}$ Chimakurthi, S. K., Tang, J., Palacios, R., Cesnik, C. E. S., and Shyy, W., "Computational Aeroelasticity Framework for
} 
Analyzing Flapping Wing Micro Air Vehicles," 49 ${ }^{\text {th }}$ AIAA/ASME/ASCE/AHS/ASC Structures, Structural Dynamics, and Materials Conference, Schaumburg, Illinois, April 2008, AIAA Paper Number 2008-1814.

${ }^{19}$ Chimakurthi, S. K., Stanford, B. K., Cesnik, C. E. S., and Shyy, W., "Flapping Wing CFD/CSD Aeroelastic Formulation Based on a Co-rotational Shell Finite Element Formulation," 50 ${ }^{t h}$ AIAA/ASME/ASCE/AHS/ASC Structures, Structural Dynamics, and Materials Conference, Palm Springs, California, May 2009, AIAA Paper Number 2009-2412.

${ }^{20}$ Gogulapati, A., Friedmann, P., and Shyy, W., "Approximate Aeroelastic Analysis of Flapping Wings in Hover," International Forum for Aeroelasticity and Structural Dynamics, No. IFASD Paper Number 2009-143, Seattle, WA, June 22-25 2009.

${ }^{21}$ MSC. MARC, Volumes $A-D, 2005$.

${ }^{22}$ Willmott, A. P. and Ellington, C. P., "The Mechanics of Flight in the Hawkmoth Manduca Sexta: I. Kinematics of Hovering and Forward Flight," The Journal of Experimental Biology, Vol. 200, 1997, pp. 2705 - 2722.

${ }^{23}$ Leishman, J. G., Principles of Helicopter Aerodynamics, Cambridge University Press, 1st ed., 2001.

${ }^{24}$ Sane, S. P., "Induced Airfoil in Flying Insects: I. A Theoretical Model of the Induced Flow," Journal of Experimental Biology, Vol. 209, 2006, pp. 32-42.

${ }^{25}$ Sane, S. P., "Induced Airfoil in Flying Insects: II. Measurement of Induced Flow," The Journal of Experimental Biology, Vol. 209, 2006, pp. 43-56.

${ }^{26}$ Katz, J. and Plotkin, A., Low-Speed Aerodynamics, Cambridge University Press, 2001.

${ }^{27}$ Bathe, K., Ramm, E., and Wilson, E. L., "Finite Element Formulations for Large Deformation Dynamic Analysis," International Journal for Numerical Methods in Engineering, Vol. 9, 1975, pp. 353 - 386.

${ }^{28}$ Bathe, K., Finite Element Procedures, Prentice-Hall Inc., 1996.

${ }^{29} \mathrm{Wu}$, P., Ifju, P., Stanford, B., Sallstrom, E., Ukeiley, L., Love, R., and Lind, R., "A Multidisciplinary Experimental Study of Flapping Wing Aeroelasticity in Thrust Production," 50th AIAA/ASME/ASCE/AHS/ASC Structures, Structural Dynamics, and Materials Conference, No. AIAA-2009-2413, Palm Springs, California, 4-7 May 2009.

${ }^{30}$ Aono, H., Chimakurthi, S., Wu, P., Sallstrom, E., Stanford, B., Cesnik, C., Ifju, P., Ukeiley, L., and Shyy, W., "A Computational and Experimental Study of Flexible Flapping Wing Aerodynamics," 48th AIAA Aerospace Sciences Meeting Including the New Horizons Forum and Aerospace Exposition, No. AIAA 2010-554, Orlando, Florida, 4-7 January 2010. 


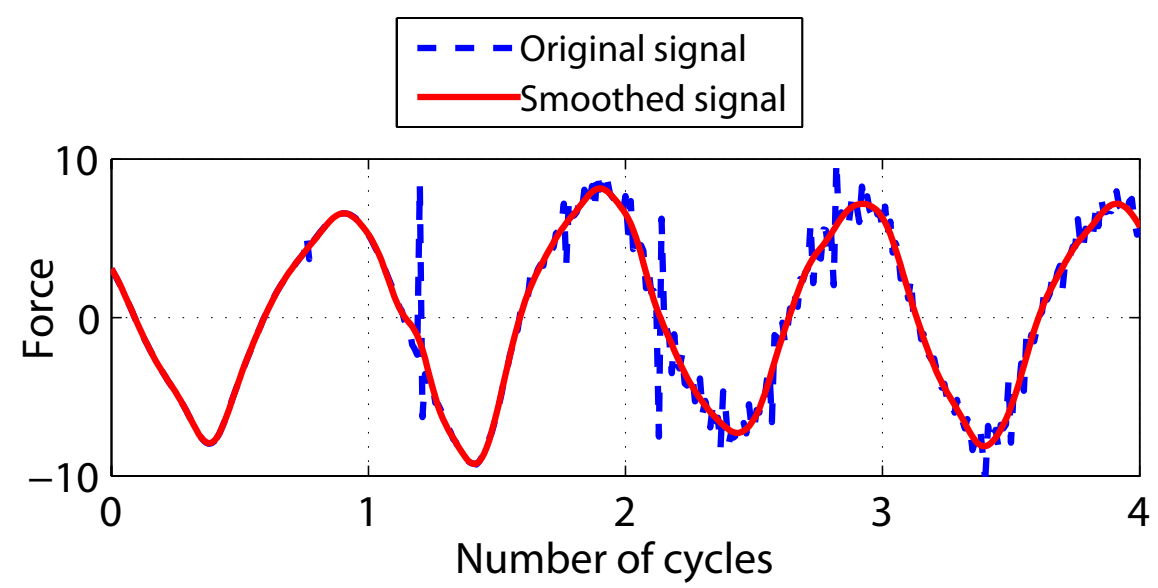

Figure 10. Sample comparison of original and filtered load signals

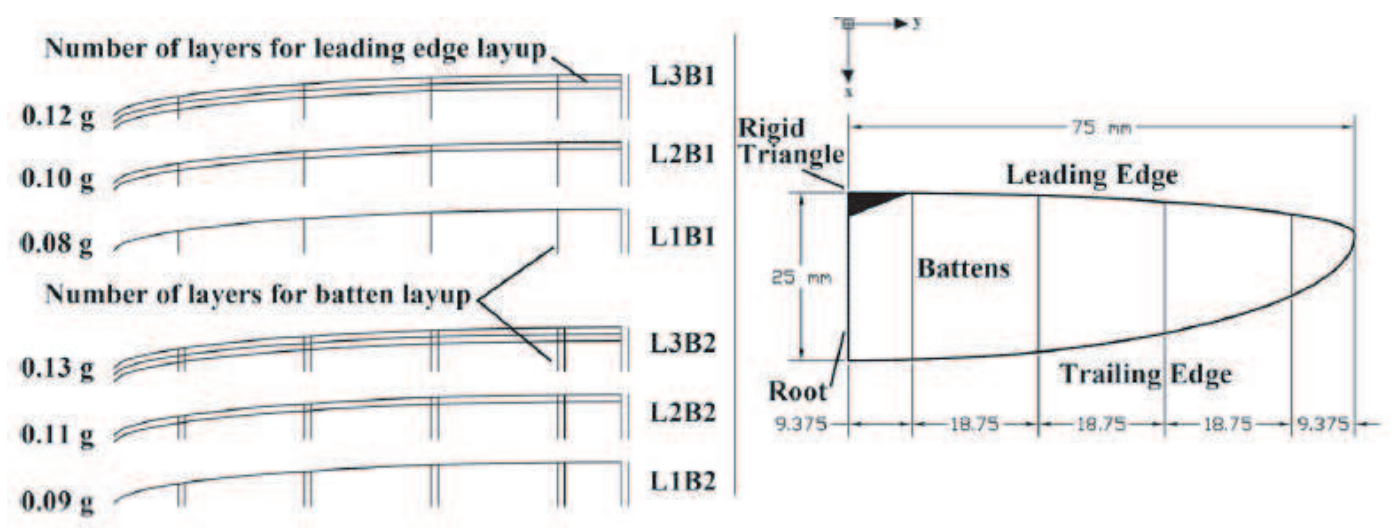

Figure 11. Anisotropic wing configurations, from Ref [29].
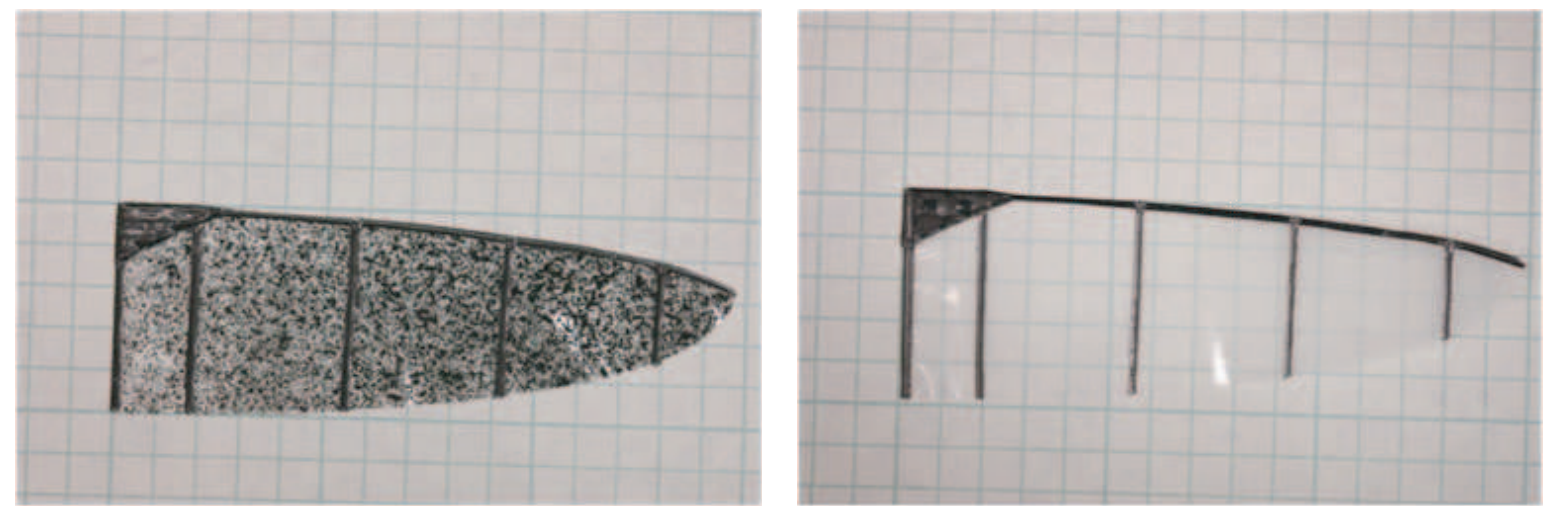

Figure 12. Painted (left) and unpainted wings (right). 


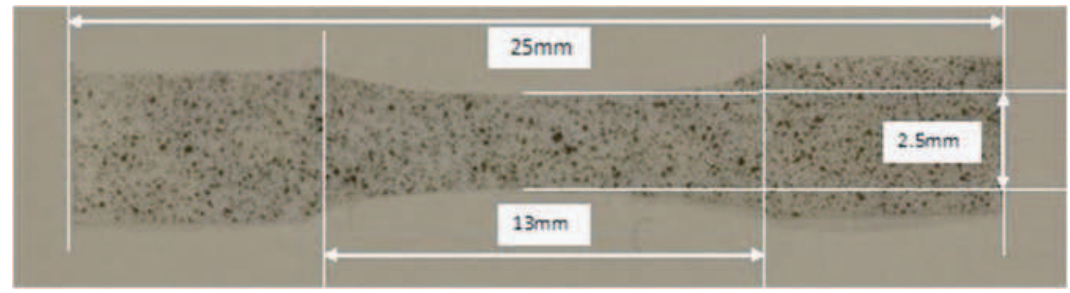

Figure 13. Tensile test specimens.

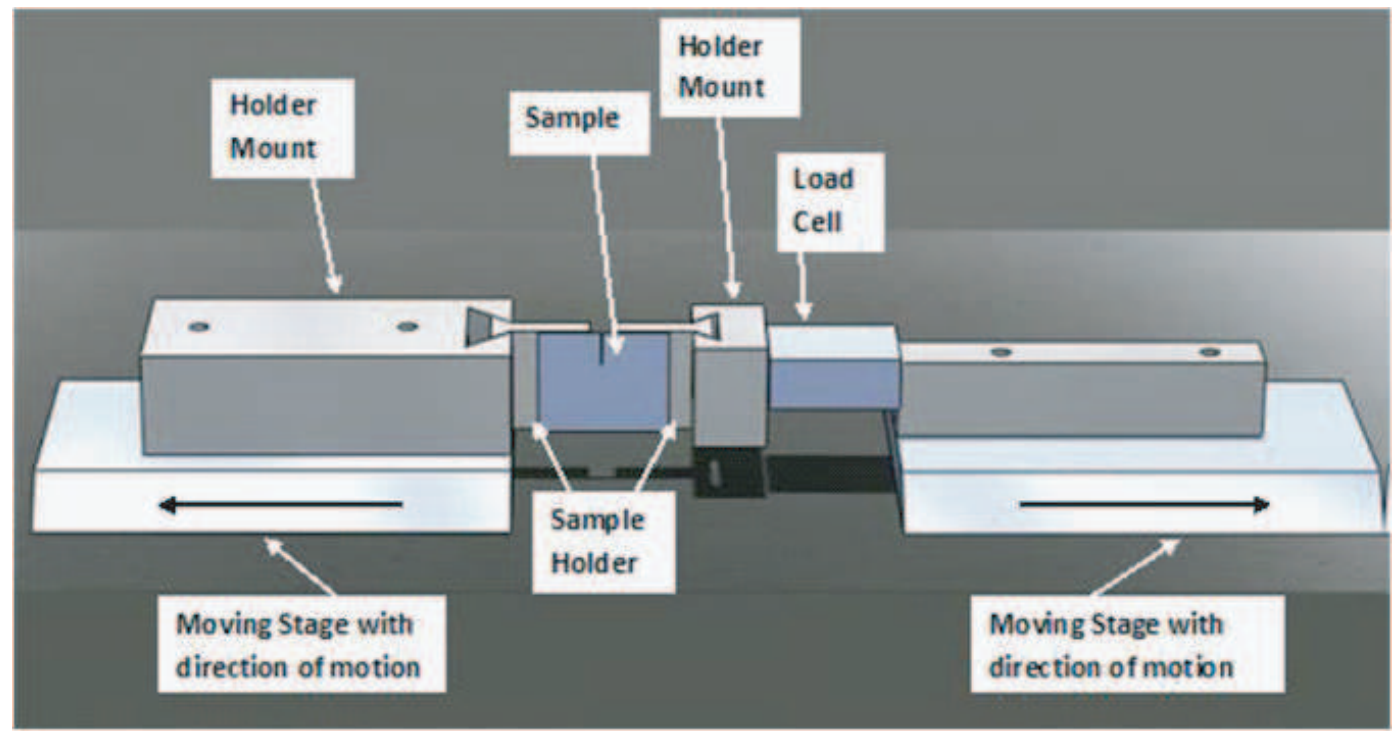

Figure 14. Experimental setup.
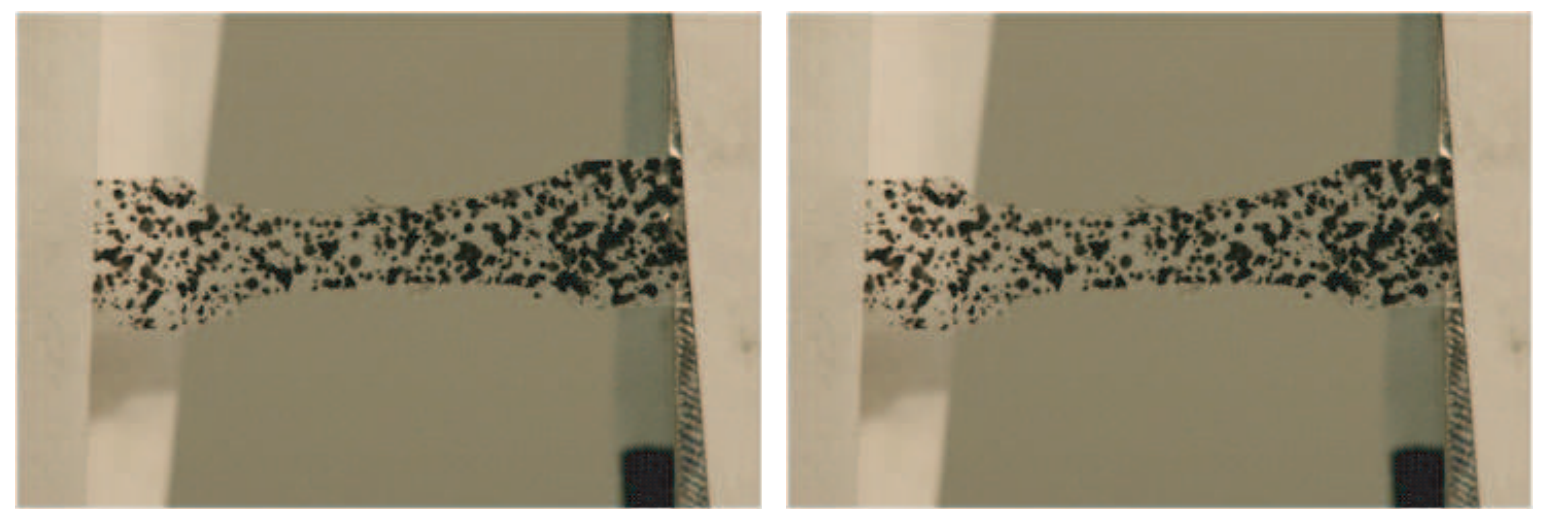

Figure 15. Specimens of the film. Dense speckle (heavy dots) - left and light speckle (light dots) - right. 


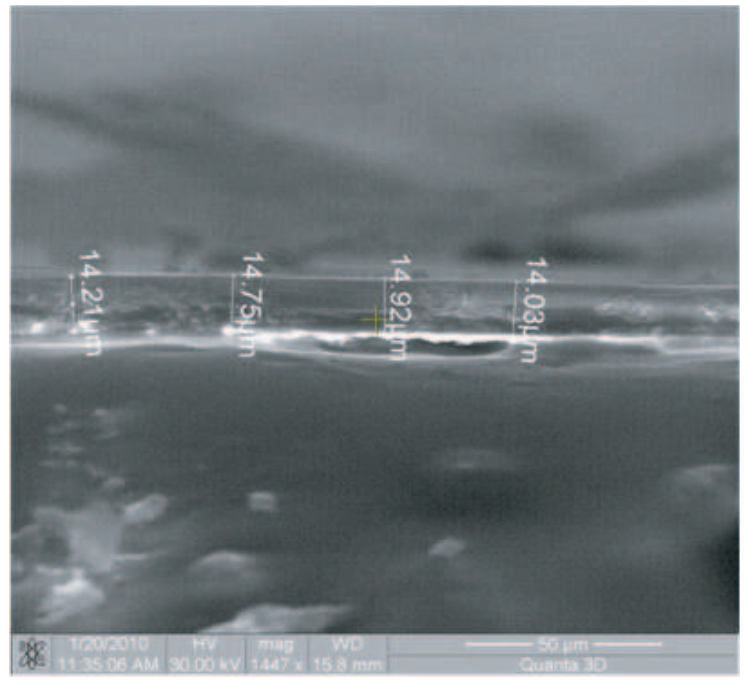

Figure 16. Sample image from the SEM indicating thickness of the film.

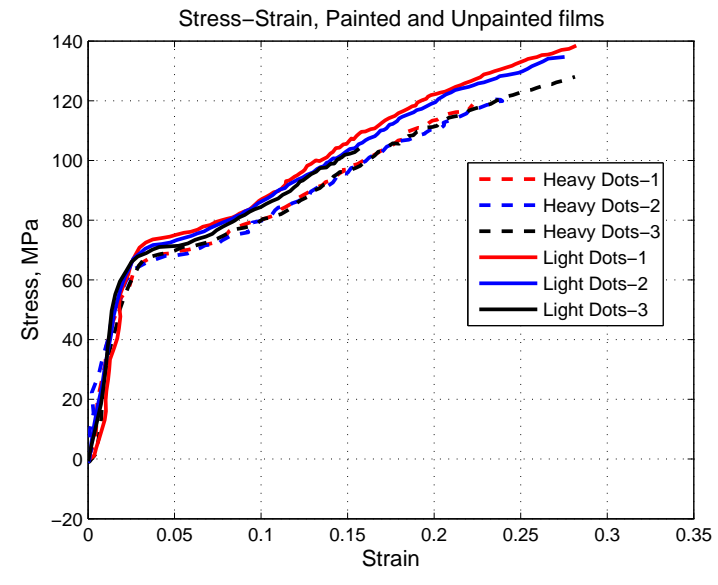

(a) Experimental stress-strain curves

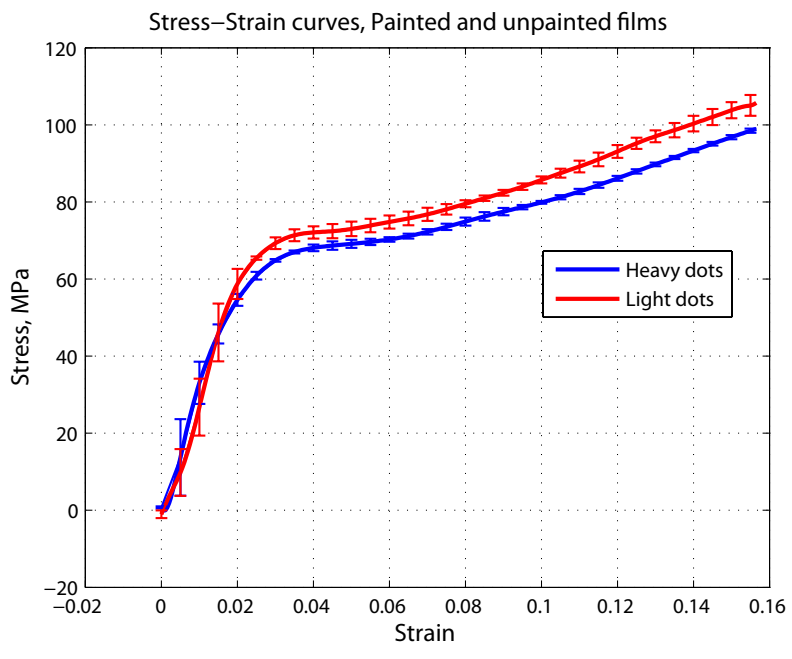

(b) Mean stress-strain curves

Figure 17. Stress-strain curves for the painted (heavy dots) and unpainted (light dots) films. 

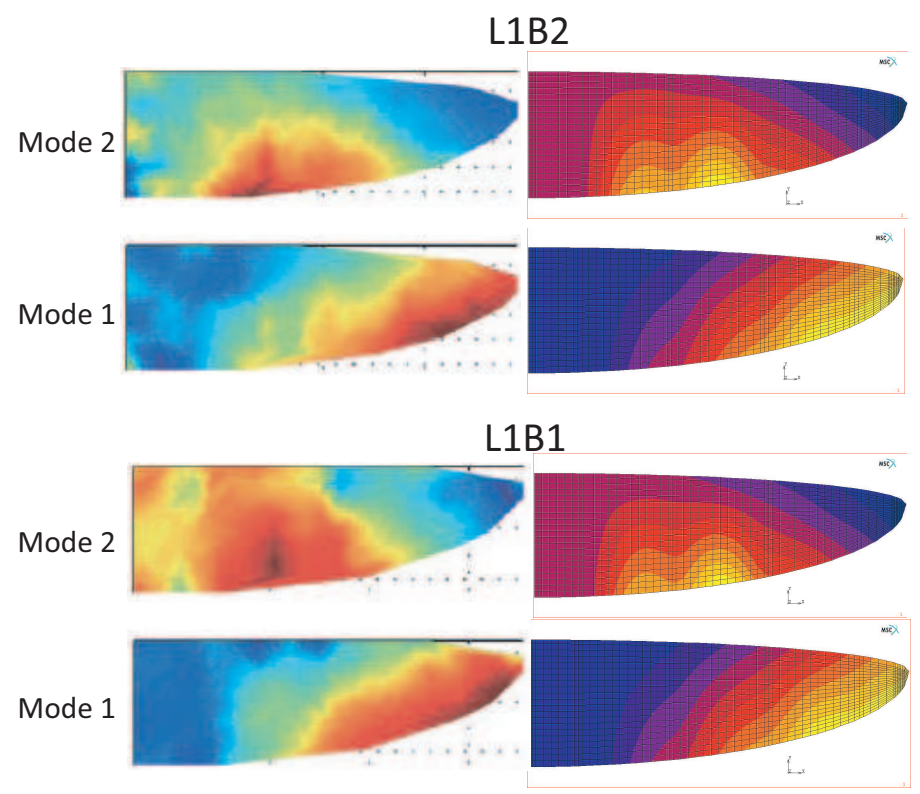

Figure 18. Comparison of mode shapes: Experiment $^{29}$ (left) and FE model - current study (right)

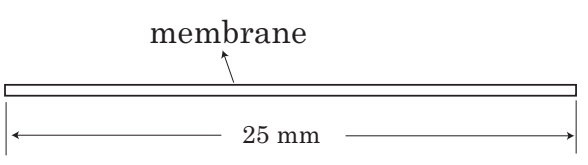

Cross-section composed of membrane

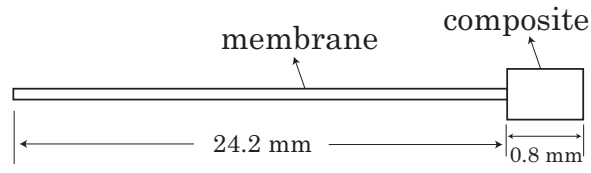

Realistic anisotropic cross-section

Figure 19. Cross sections used to compute mass ratios for the anisotropic wings. 


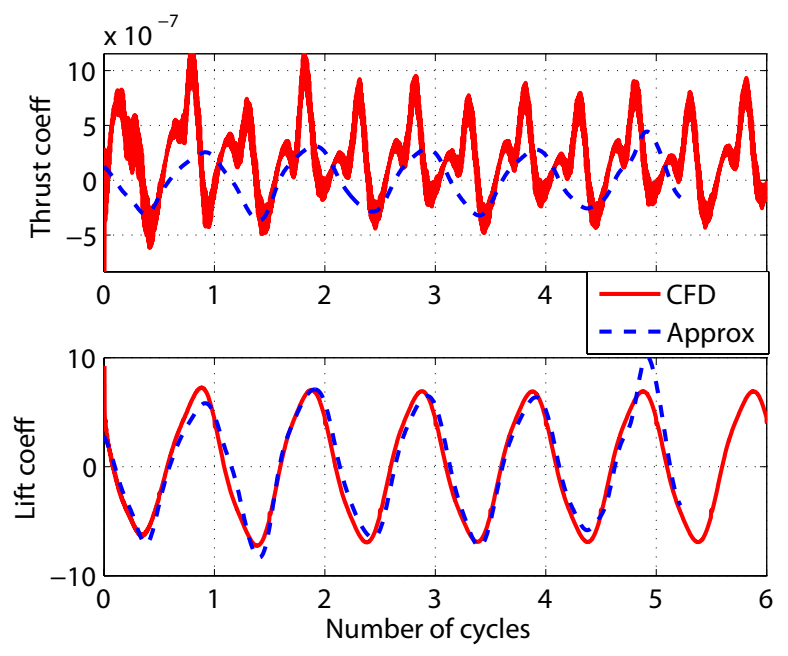

(a) Rigid
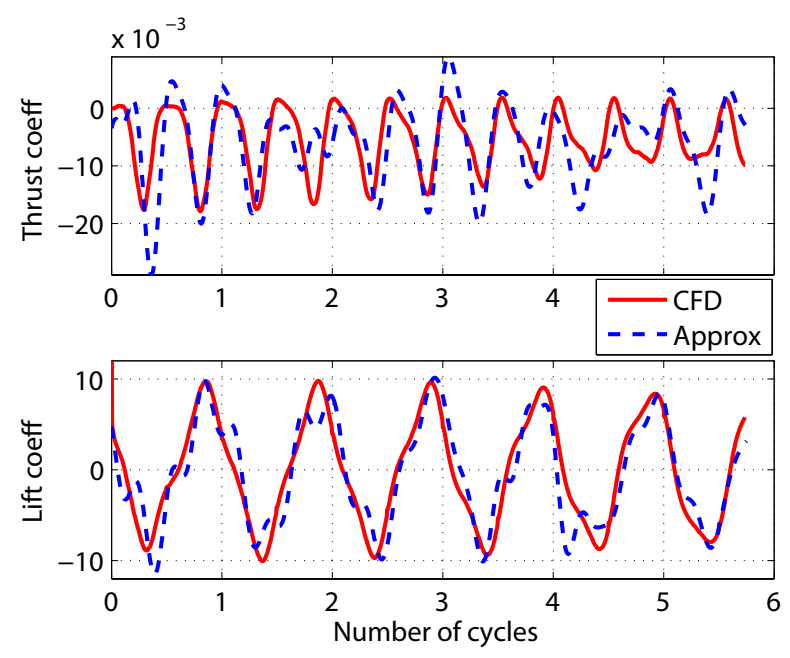

(c) $\mathrm{E}=10 \mathrm{GPa}$
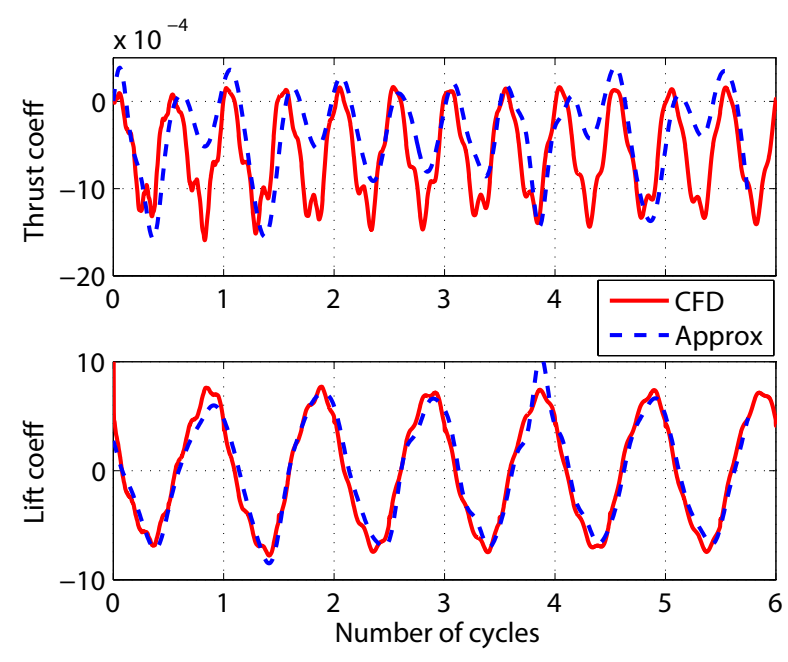

(b) $\mathrm{E}=70 \mathrm{GPa}$
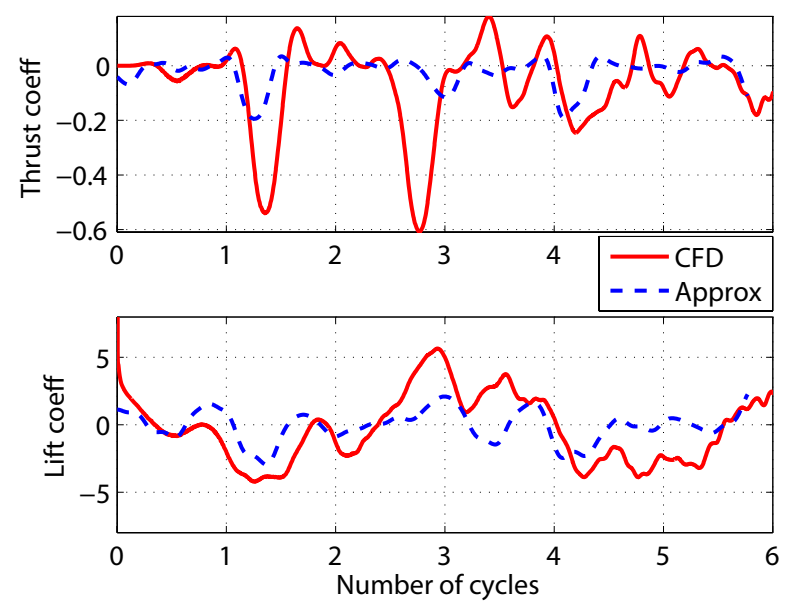

(d) $\mathrm{E}=0.1 \mathrm{GPa}$

Figure 20. Force coefficients generated by rigid and flexible wings undergoing prescribed flapping motion 


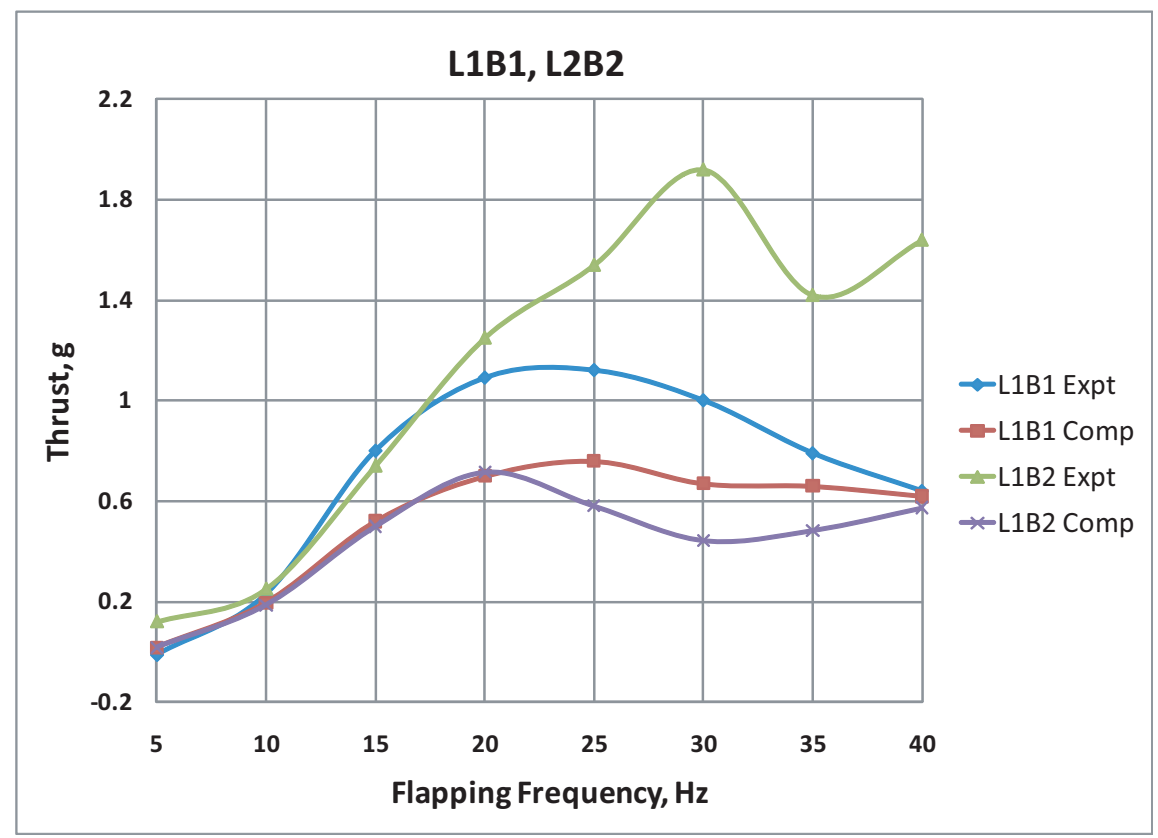

Figure 21. Thrust generated by L1B1 and L1B2: 'Expt' - Experiments, ${ }^{29}$ 'Comp' - computations, current study

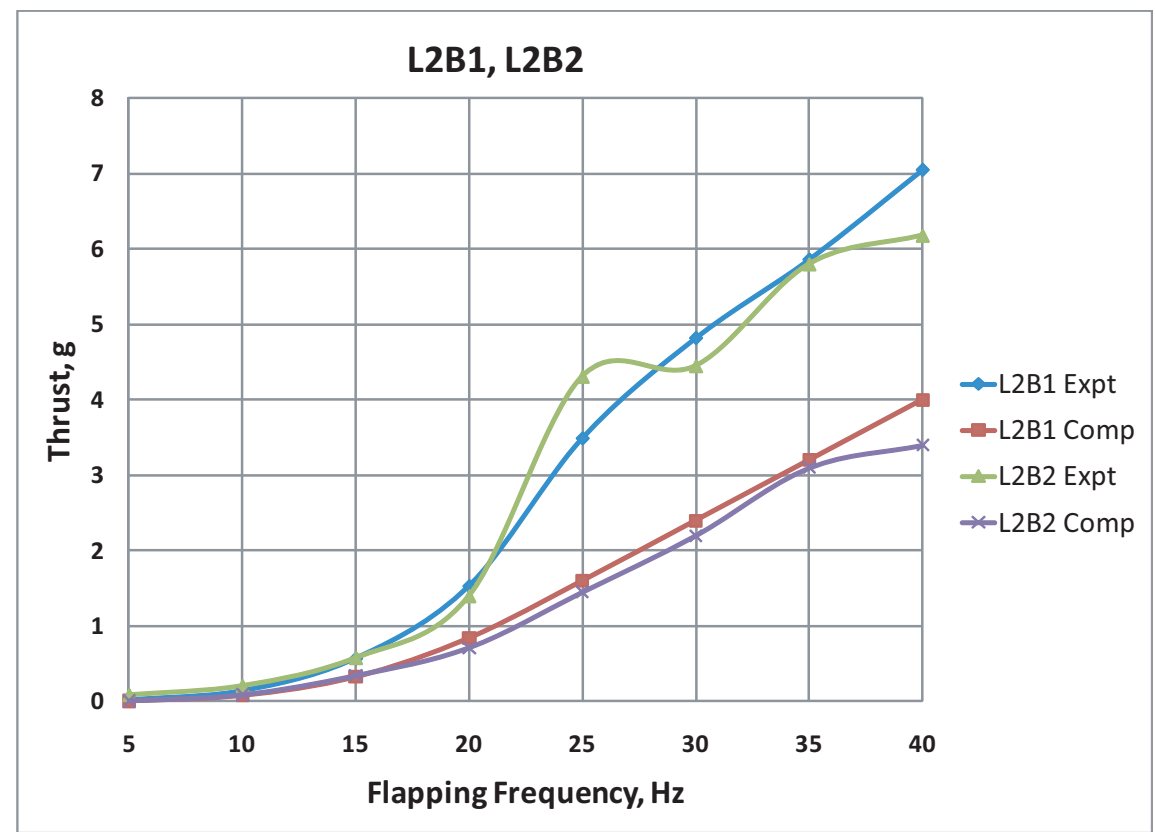

Figure 22. Thrust generated by L2B1 and L2B2: 'Expt' - Experiments, ${ }^{29}$ 'Comp' - computations, current study 


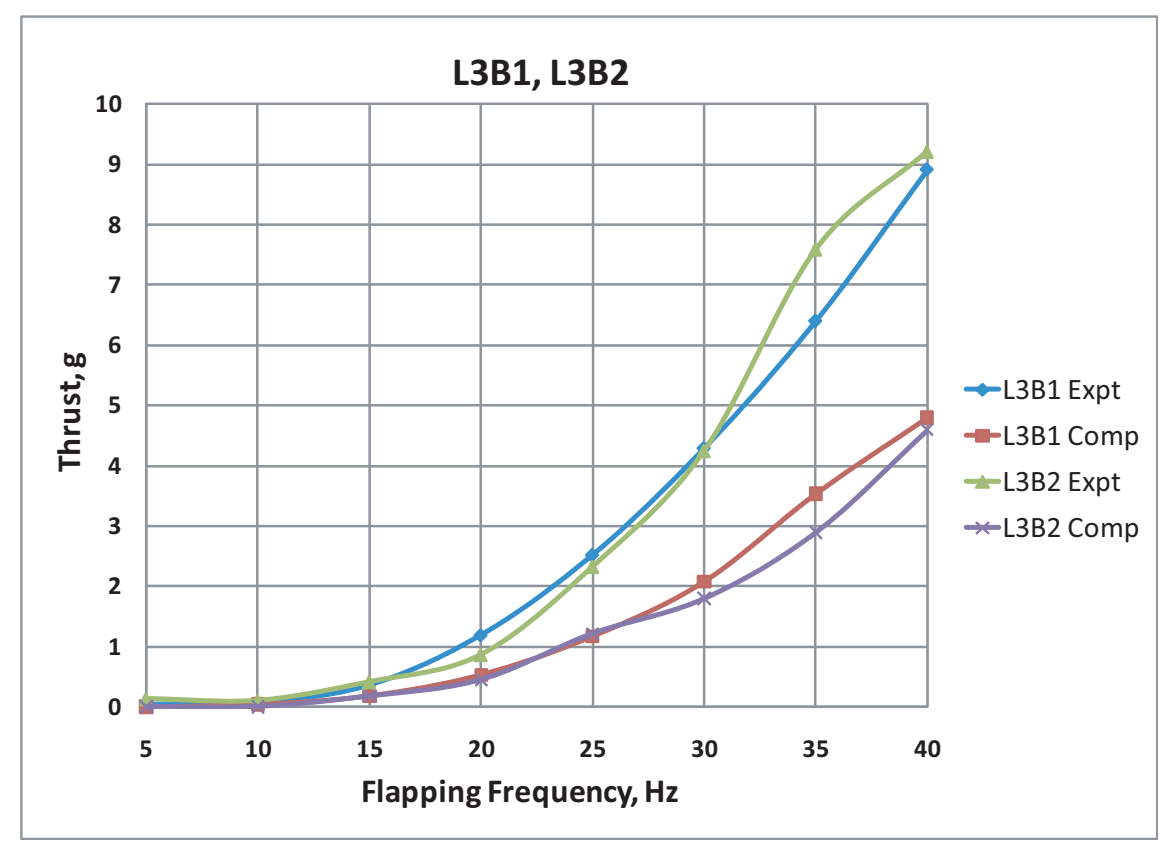

Figure 23. Thrust generated by L3B1 and L3B2: 'Expt' - Experiments, ${ }^{29}$ 'Comp' - computations, current study
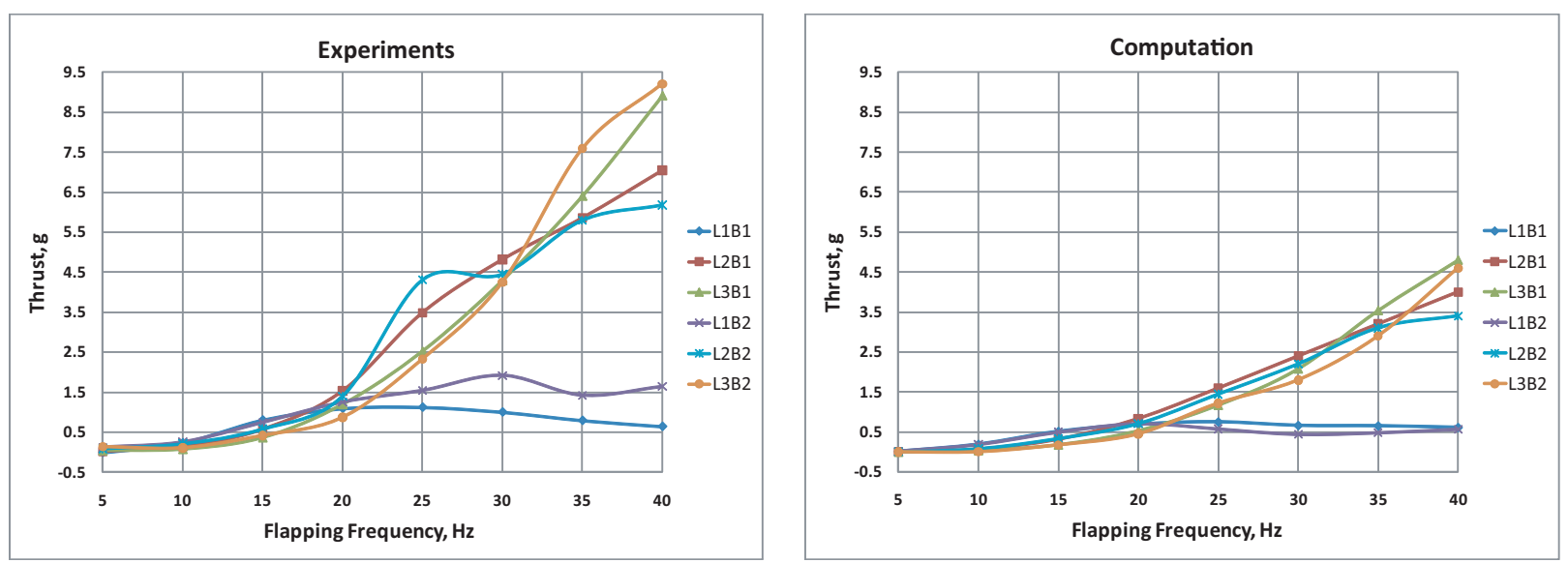

Figure 24. Thrust generated by all configurations: Experiment ${ }^{29}$ (left), Computation (right) 


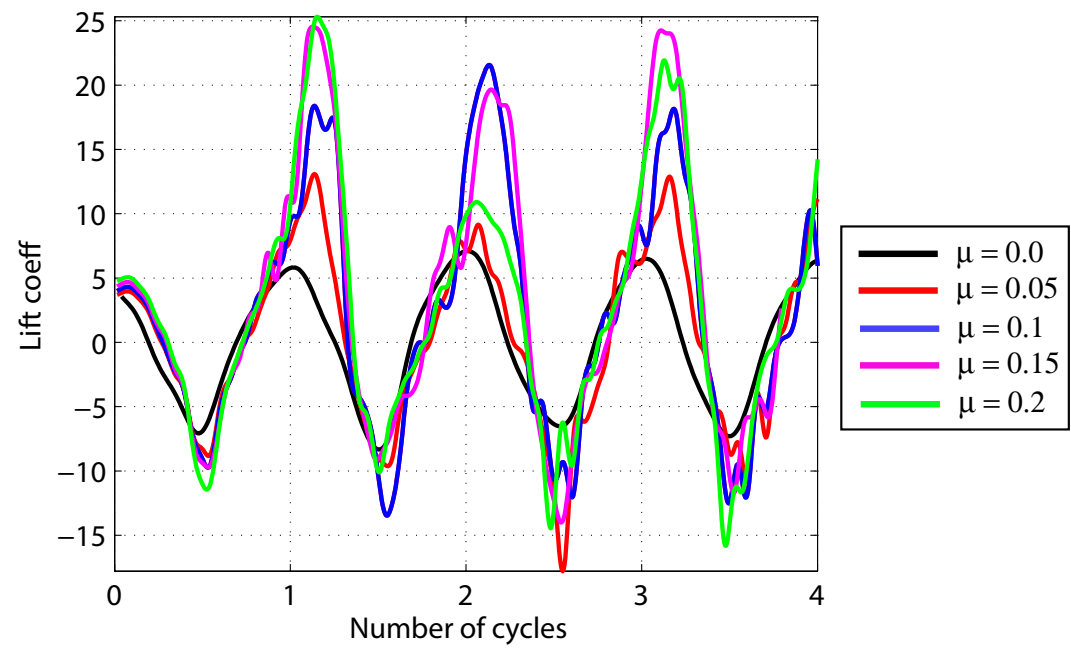

Figure 25. Comparison of lift coefficients for a rigid flapping wing for various advance ratios 